\author{
Universidade de São Paulo \\ Instituto de Física
}

\title{
Modelos para sitemas quânticos abertos com aplicações em portas lógicas holonômicas e transporte quântico
}

\section{Pedro Vinicius de Castro Portugal}

Orientador: Prof. Dr. Gabriel Teixeira Landi.

Dissertação de mestrado apresentada ao Instituto de Física como requisito parcial para a obtenção do título de Mestre em Ciências.

Banca Examinadora:

Prof. Dr. Gabriel T. Landi (Instituto de Física da Universidade de São Paulo)

Prof. Dr. Frederico B. Brito (Instituto de Física de São Carlos da Universidade de São Paulo)

Prof. Dr. Raphael C. Drumond (Universidade Federal de Minas Gerais) 


\section{FICHA CATALOGRÁFICA \\ Preparada pelo Serviço de Biblioteca e Informação \\ do Instituto de Física da Universidade de São Paulo}

Portugal, Pedro Vinicius Castro

Modelos para sistemas quânticos abertos com aplicações em portas lógicas holonômicas e transporte quântico. São Paulo, 2019.

Dissertação (Mestrado) - Universidade de São Paulo. Instituto de Física. Depto. de Física dos Materiais e Mecânica.

Orientador: Prof. Dr. Gabriel Teixeira Landi

Área de Concentração: Física da Matéria Condensada

Unitermos: 1. Informação quântica; 2. Física teórica; 3. Sistema quântico. 
University of São Paulo

Physics Institute

\section{Models for open quantum systems with applications to holonomic gates and quantum transport}

\section{Pedro Vinicius de Castro Portugal}

Supervisor: Prof. Dr. Gabriel Teixeira Landi.

Dissertation submitted to the Physics Institute of the University of São Paulo in partial fulfillment of the requirements for the degree of Master of Science.

Examining Committee:

Prof. Dr. Gabriel T. Landi (Physics Institute of the University of São Paulo)

Prof. Dr. Frederico B. Brito (São Carlos Physics Institute of the University of São Paulo)

Prof. Dr. Raphael C. Drumond (Federal University of Minas Gerais) 
"The interpretation of quantum mechanics has been dealt with by many authors, and I do not want to discuss it here. I want to deal with more fundamental things." — Paul Dirac. 


\section{Acknowledgments}

I would like to acknowledge my parents for the emotional and financial support without which this would not be possible. I would especially like to acknowledge my mother for her unconditional patience and unwavering support.

I would like to acknowledge Gabriel Landi's exceptional supervision. I can't remember Landi not being willing to discuss anything about physics. His interest and competence are inspiring. I thank Jader for the useful and frequent discussions.

I would like to acknowledge Erik Sjöqvist for welcoming be in Uppsala and advising me in the interesting topic of holonomic quantum computation.

I would also like to acknowledge Pedro Harunari, who has been my friend for a long time, and will always be an inspiration of what a physicist should be. I thank you in retrospect and in advance for helping me with revision and bureaucracies related to this dissertation.

I acknowledge CNPq and ERASMUS for the financial support. 


\begin{abstract}
Open quantum systems play a major role in many field of physics since quantum systems are generally very hard to screen from the environment, which makes noise and decoherence a major setback in quantum technologies such as quantum computers.

In this dissertation we carry out a study of open quantum systems in several paradigms: in the context of holonomic quantum computation, where it is shown that the environment can improve the implementation of a quantum gate; in the context of transport in a Bosonic chain, where some entropic quantities are calculated; in the context of quantum to classical transition, where quantum darwinism is characterized in an exactly soluble model; and in the study of an exact solution to a Fermionic model, using non-equilibrium Green's functions.
\end{abstract}

Keywords: Open quantum systems; Holonomic gates; Quantum information; Entropy production; Non equilibrium Green's functions 


\section{Resumo}

Os sistemas quânticos abertos tem um papel importante em muitos campos da física, uma vez que os sistemas quânticos são geralmente muito difícil de isolar do ambiente, o que torna o ruído e a descoerência um dos principais reveses em tecnologias quânticas, como computadores quânticos.

Nesta dissertação, realizamos um estudo de sistemas quânticos abertos em vários paradigmas: no contexto da computação quântica holonômica, onde é mostrado que o ambiente pode melhorar a implementação de uma porta lógica quântica; no contexto do transporte em uma cadeia bosônica, onde algumas quantidades entrópicas são calculadas; no contexto de transição quântica para clássica, onde o darwinismo quântico é caracterizado em um modelo exatamente solúvel; e no estudo de uma solução exata para um modelo Fermionico, usando as funções de Green de não equilíbrio.

Palavras chave: Sistemas quânticos abertos; Portas lógicas holonômicas; Informação quântica; Produção de entropia; Funções de Green de não equilíbrio. 


\section{Contents}

\begin{tabular}{|lll}
\hline & Introduction & 6
\end{tabular}

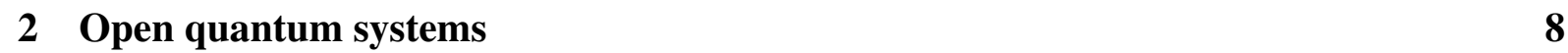

$2.1 \quad$ Introduction to density matrix theory $\ldots \ldots \ldots \ldots$. . . . . . . . . 8

$2.1 .1 \quad$ Lindblad's theorem . . . . . . . . . . . . . . . . . . . . 9

2.2 General method for deriving a master equation . . . . . . . . . . . 10

$2.2 .1 \quad$ Physically motivated microscopic derivation $\ldots \ldots \ldots$

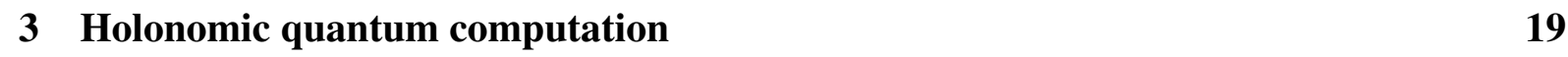

$3.1 \quad$ Introduction $\ldots \ldots \ldots \ldots \ldots$

3.2 Holonomic quantum gates $\ldots \ldots \ldots \ldots$. . . . . . . . . . 20

3.2.1 Berry's phase and Wilczek-Zee phase . . . . . . . . . . . 20

3.2 .2 Non-adiabatic holonomic quantum gates . . . . . . . . . . . . 25

3.3 Environment assisted implementation . . . . . . . . . . . . . 27

$3.3 .1 \quad$ Initial states and pulse error $\ldots \ldots \ldots \ldots . \ldots \ldots$

3.3 .2 Master equation . . . . . . . . . . . . . . . 28

3.3 .3 Markovian limit noise . . . . . . . . . . . . . . 28

3.3 .4 Including a relaxation time . . . . . . . . . . . . . . . 29

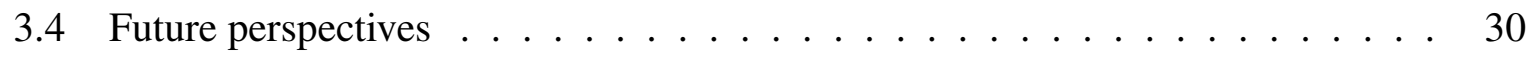

4 Thermodynamics of Bosonic Gaussian states 32

$4.1 \quad$ Wigner representation and quantum Gaussian states . . . . . . . . . . . . 32

$4.2 \quad$ Wigner entropy $\ldots \ldots \ldots \ldots \ldots$. . . . . . . . . . . . . . . . . . . 34

4.3 Quantum Fokker-Plank equation $\ldots \ldots \ldots \ldots$. . . . . . . . . . 35

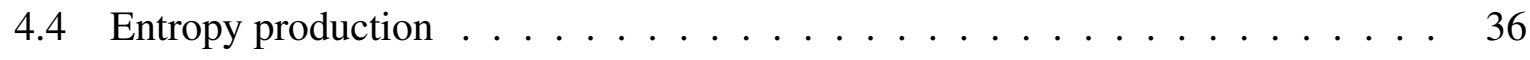

4.5 Wigner entropy production $\ldots \ldots \ldots \ldots \ldots \ldots$ 
5 Thermodynamics and information in a bosonic chain 39

5.1 A note on global vs. local master equations $\ldots \ldots$. . . . . . . . . . . 39

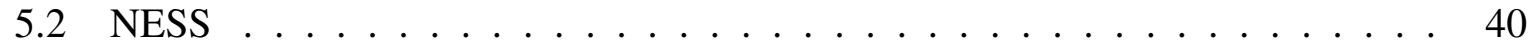

5.3 Notes on the reduced covariance matrix f . . . . . . . . . . . . . 41

5.4 Tridiagonal Toeplitz matrix and Chebishev Polynomials . . . . . . . . . . . . 42

5.5 Covariance like, toeplitz like matrix determinant . . . . . . . . . . . . 43

5.6 Mutual information and entropy production $\ldots \ldots \ldots$. . . . . . . . . 43

6 Quantum Darwinism 45

6.1 Model and time evolution . . . . . . . . . . . . . . . . . . . 45

6.2 Measure-and-prepare maps . . . . . . . . . . . . . . . . . . . 48

6.3 Numerical analysis of the problem $\ldots \ldots \ldots \ldots$. . . . . . . . 50

6.4 Bath modeling . . . . . . . . . . . . . . . . . . . 50

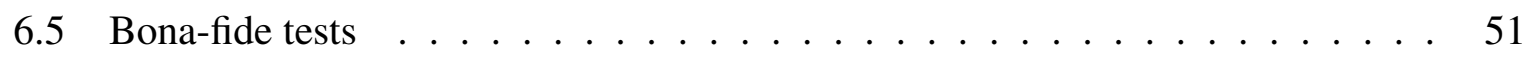

6.6 Conclusions and perspectives $\ldots \ldots \ldots \ldots \ldots \ldots$. . . . . . . . . . 52

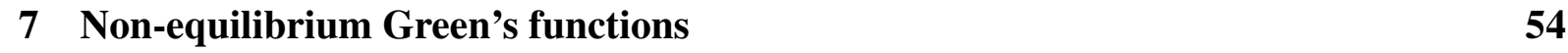

7.1 Model . . . . . . . . . . . . . . . . . . . . . . . . . . . . . 54

7.2 Correlations . . . . . . . . . . . . . . . . . 56

7.3 Lyapunov equation $\ldots \ldots \ldots \ldots \ldots \ldots \ldots$

7.3 .1 Lindblad equation $\ldots \ldots \ldots \ldots \ldots$

7.4 Bath acting on a single site $\ldots \ldots \ldots \ldots \ldots$. . . . . . . . . . . . 59

7.4.1 Finite temperature and characteristic distances $\ldots \ldots$. . . . . . 60

7.4.2 Time evolution of noise-system correlations . . . . . . . . . . . . . 60

7.4 .3 Infinite time correlations . . . . . . . . . . . . . . 62

7.4 .4 Chemical potential . . . . . . . . . . . . . . 62

$7.4 .5 \quad$ Additivity . . . . . . . . . . . . . . . . . 63

\begin{tabular}{ll|r}
\hline 8 & Conclusion & 64
\end{tabular} 


\section{Chapter 1}

\section{Introduction}

Some problems that look simple can be very hard to describe. Take for example a particle in a cup of water. An unimaginable number of water molecules collide with it and with each other with really complicated interactions, which would be unimaginable to solve with Newton's laws. That said, when we look at such molecule the behaviour cannot be that complicated. It jiggles, and if it is moved it looses speed and goes back to jiggling. With that in mind there must be a way to describe it without having to solve a $10^{23}$ variables equation.

It turns out that there is a field in physics, called stochastic physics which deals with this type of problem [1]. It solves this problem of having to deal with the complexity of the environment, in the example the water, by assuming that the interactions produce a random movement.

This idea of only looking at the system and trying to describe the effects of a very complicated environment in simpler terms also extends to quantum mechanics, in the field of open quantum systems, though the approach taken is not so straightforward. A series of equations are used to describe a system in contact with an environment, such as Lindblad equations [2, 3], quantum Fokker-Plank equations [4] and also some stochastic methods such as quantum jumps [5, 6, 7]. In this dissertation some of these approaches will be described, especially Lindblad equations and quantum Fokker-Plank equations.

This dissertation will include three types of problems where open quantum systems play major, but different, roles.

The first problem is an application of open quantum systems in quantum computation, more specifically to a holonomic quantum gate.

Two transport problems will be studied as well. One in a Bosonic chain, where concepts of quantum information will be discussed. Then in a Fermionic chain, where an analytic solution 
of the problem is feasible and detailed discussions on the basics of open quantum systems are possible.

In chapter 6 the quantum to classical transition is discussed in the context of quantum Darwinism. 


\section{Chapter 2}

\section{Open quantum systems}

\subsection{Introduction to density matrix theory}

Density matrices are a powerful tool to describe loss of information to the environment. By definition, they allow description of quantum states with a classical ignorance, by adding a classical probability to a quantum state. They are defined as positive matrices with unit trace, and can be written as

$$
\rho=\sum_{i} p_{i}\left|\psi_{i}\right\rangle \psi_{i} \mid
$$

where $p_{i}$ are probabilities. One can show that averages of operators can be calculated with $\langle A\rangle=\operatorname{tr}[A \rho]$, and that a unitary time evolution is given by $\dot{\rho}=-i[H, \rho]$, known as the vonNeumann equation.

Density matrices also appear naturally when considering subsystems. For example, take two entangled qubits in the state $\left|\psi_{A B}\right\rangle=(|00\rangle+|11\rangle) / \sqrt{2}$. If only one of the qubits is measured in any bases the two possible outcomes will have equal probabilities, meaning the state of qubit A cannot be pure. Instead it can be described by the density matrix

$$
\rho_{A}=\mathbb{I} / 2,
$$

which is bases independent, and also correctly describes the 50-50 probabilities in all bases.

Formally, the concept of measurement can be generalized with a set $M_{i}$ such that $\sum_{i} M_{i}^{\dagger} M_{i}=$ I. The outcomes $i$ and probabilities associated with such measurement will be given by $p_{m}=$ $\operatorname{tr}\left(M_{i}^{\dagger} M_{i} \rho\right)$. The equivalent of the wavefunction collapse is $\rho_{i}=\frac{M_{i} \rho M_{i}^{\dagger}}{p_{i}}$. These measurements naturally include projective measurements when $M_{i}$ is a set of projectors composed of outer 
products of an orthonormal bases.

One key concept in density matrix theory is the purity of a quantum state, defined as $P[\rho]=$ $\operatorname{tr}\left[\rho^{2}\right]$. It is 1 if the state is pure and attains its lowest value, $1 / d$ (where $d$ is the dimension of the Hilbert space) if the state is maximally mixed. Incidentally this is a measure of how entangled the subsystem is to the other part (assuming the composite state is pure) [8], as in the example above, a maximally entangled state resulted in a maximally mixed subsystem, with purity $1 / 2$. This alone can be used to argue that to get a pure state to become mixed it is necessary to entangle it to another quantum system, which shows that entanglement plays a crucial role in open quantum systems

Just like a subsystem cannot necessarily be described by a pure state, when a system is open its dynamics will not necessarily be unitary. Instead it is a general evolution that takes positive matrices with unit trace to positive matrices with unit trace at all times, continuously, known as Completely-Positive-Trace-Preserving(CPTP) dynamical maps [10].

A positive map is a linear map that takes positive matrices to positive matrices, but this is not enough for a map to be physically acceptable. Complete positivity implies not only that a map $M$ is positive, but $M \otimes \mathbb{I}$ is also positive [11], where $\mathbb{I}$ is the identity in any dimension. This is necessary since the map $M \otimes \mathbb{I}$ is simply applying $M$ and doing nothing in an arbitrary system, which should still be positive.

\subsubsection{Lindblad's theorem}

Since physical maps are CPTP, it would be interesting to have the most general form of dynamical CPTP maps. Unfortunately, there isn't one yet ${ }^{2}$, One very useful theorem in that regard is Lindblad's theorem. It concerns evolutions that have the semi-group property

$$
\exp \left\{\left(t-t_{0}\right) \mathcal{L}\right\}\left[\rho\left(t_{0}\right)\right]=\rho(t)
$$

\footnotetext{
${ }^{1}$ A recent article argues that one can obtain decoherence without entanglement [9], but it assumes a classical source of randomness, in some sense using a new ensemble. In particular, if the classical noise in said paper comes from a quantum environment, the system entangles with it.

${ }^{2}$ There is a general form for CPTP maps, known as Kraus maps [12, 13], but this does not imply there is a closed form for the family of CPTP maps on all times for a dynamical map. For example, a Linblad like equation with negative coefficients can be positive, as will be seen in chapter 7 .
} 
where $\mathcal{L}$ is a constant generator. The theorem states that if the generator has the form [14]

$$
\begin{array}{r}
\mathcal{L}[\rho]=-\mathrm{i}[H, \rho]+\sum_{i} \gamma_{i} \mathrm{D}\left[a_{i}, a_{i}^{\dagger}, \rho\right] \\
\mathbb{D}\left[a_{i}, a_{i}^{\dagger}, \rho\right]=a_{i} \rho a_{i}^{\dagger}-\frac{1}{2}\left\{a_{i}^{\dagger} a_{i}, \rho\right\}
\end{array}
$$

where $a_{i}$ are arbitrary operators in the Hilbert space, $H$ is Hermitian and $\gamma_{i} \geq 0$ then the dynamical map $\exp \left\{\left(t-t_{0}\right) \mathcal{L}\right\}$ is CPTP. This is a good starting point for Markovian maps, which are defined as $\mathrm{CP}$ divisible maps with the property

$$
\Lambda_{t, t_{0}}=\Lambda_{t, t_{1}} \Lambda_{t_{1}, t_{0}}
$$

for $t>t_{1}>t_{0}$, where $\Lambda_{t_{i}, t_{j}}$ is CPTP for all $t_{i}>t_{j}$. Maps that form semigroups satisfy this property, but there are more general maps that do not.

A generalization of Lindblad's theorem states that maps are CP divisible if and only if they can be cast in the form

$$
\mathcal{L}[\rho]=-\mathrm{i}[H, \rho]+\sum_{i} \gamma_{i}(t) \mathbb{D}\left[a_{i}(t), a_{i}^{\dagger}(t), \rho\right]
$$

with $\gamma_{i}(t) \geq 0$ for all t. Maps like this are common in microscopic derivations, though sometimes $\gamma$ is negative as will be seen in this chapter and in chapter 7.

If $\gamma(t)$ is not always positive then the map is called non-Markovian. Non-Markovianity is deeply linked with backflow of information [15] so it plays many roles in quantum information.

\subsection{General method for deriving a master equation}

In this section we introduce a general method for deriving master equations, based on the projection operator technique of Nakajima and Zwanzig [16, 17].

Supose a system $S$, with Hamiltonian $H_{S}$, interacts with an environment $E$, with Hamiltonian $H_{E}$, via an interaction Hamiltonian $\alpha H_{I}$, where $\alpha$ is just a bookkeeping parameter introduced for convenience. The von Neumann equation for the evolution of the joint density matrix of $S$ and $E$, in the interaction picture, will be

$$
\frac{\partial}{\partial t} \rho(t)=-i \alpha\left[H_{I}(t), \rho(t)\right] \equiv \alpha \mathcal{L}(t) \rho(t)
$$


As customary in the field of open quantum systems, we will assume that the environment is large and thus only slightly perturbed by the interaction with the system. With this in mind we define a set of projection super-operators

$$
\begin{gathered}
\rho \mapsto \mathcal{P} \rho=\operatorname{tr}_{B}\{\rho\} \otimes \rho_{B} \equiv \rho_{S} \otimes \rho_{B}, \\
Q \rho=\rho-\mathcal{P} \rho .
\end{gathered}
$$

They are indeed projection operators since they satisfy:

$$
\begin{aligned}
\mathcal{P}+Q & =I, \\
\mathcal{P}^{2} & =\mathcal{P}, \\
Q^{2} & =Q, \\
\mathcal{P} Q & =\mathcal{Q P}=0 .
\end{aligned}
$$

These projectors capture the idea that the bath is almost in the thermal state $\rho_{B} \approx e^{-\beta H_{B}} / \operatorname{tr}\left\{e^{-\beta H_{B}}\right\}$, so in order to derive an equation for the reduced system assuming the bath is in the thermal state is a good starting point. Of course the full state is not really $\rho_{S} \otimes \rho_{B}$, after all if that were the case the system would not be entangled with the bath, implying it would always be pure, which would not properly thermalize.

Each projection will follow the equations of motion

$$
\begin{aligned}
& \frac{\partial}{\partial t} \mathcal{P} \rho(t)=\mathcal{P} \frac{\partial}{\partial t} \rho(t)=\alpha \mathcal{P} \mathcal{L}(t) \rho(t)=\alpha \mathcal{P} \mathcal{L}(t) \mathcal{P} \rho(t)+\alpha \mathcal{P} \mathcal{L}(t) Q \rho(t) \\
& \frac{\partial}{\partial t} Q \rho(t)=Q \frac{\partial}{\partial t} \rho(t)=\alpha \mathcal{Q} \mathcal{L}(t) \rho(t)=\alpha \mathcal{Q} \mathcal{L}(t) \mathcal{P} \rho(t)+\alpha \mathcal{Q} \mathcal{L}(t) Q \rho(t)
\end{aligned}
$$

where in the last step the identity $\mathbb{I}=\mathcal{P}+Q$ was inserted. Since the objective is to describe the evolution of $\rho_{S}$, the projection from $Q$ does not really need a detailed solution, instead one can formally solve it by introducing a Green's function

$$
Q \rho(t)=\mathcal{G}\left(t, t_{0}\right) \mathcal{Q} \rho\left(t_{0}\right)+\alpha \int_{t_{0}}^{t} d s \mathcal{G}(t, s) \mathcal{Q} \mathcal{L}(s) \mathcal{P} \rho(s)
$$

where

$$
\mathcal{G}(t, s) \equiv T \exp \left[\alpha \int_{s}^{t} d s^{\prime} Q \mathcal{L}\left(s^{\prime}\right)\right]
$$


and $T$ is a time ordering operator. The Green's function satisfies the equation

$$
\frac{\partial}{\partial t} \mathcal{G}(t, s)=\alpha \mathcal{L}(t) \mathcal{G}(t, s)
$$

Inserting this formal solution in the $\mathcal{P}$ part results in what is known as Nakajima-Zwanzing equation.

$$
\begin{aligned}
\frac{\partial}{\partial t} \mathcal{P} \rho(t)= & \alpha \mathcal{P} \mathcal{L}(t) \mathcal{G}\left(t, t_{0}\right) \mathcal{Q} \rho\left(t_{0}\right)+\alpha \mathcal{P} \mathcal{L}(t) \mathcal{P} \rho(t) \\
& +\alpha^{2} \int_{t_{0}}^{t} d s \mathcal{P} \mathcal{L}(t) \mathcal{G}(t, s) \mathcal{Q} \mathcal{L}(s) \mathcal{P} \rho(s)
\end{aligned}
$$

Up to this point no approximations were made, not even assuming the initial state of the bath is thermal. Mathematically $\rho_{B}$ could be anything, but assuming it is the initial state of the environment makes calculations easier (and is the natural choice).

Assuming the initial condition is factorized, meaning $\rho(0)=\rho_{S}(0) \otimes \rho_{B}(0)$, the first term in eq. 2.15 is zero, because $Q \rho_{S}(0) \otimes \rho_{B}(0)=0$. The resulting equation is still not particularly easy to solve, especially because it has a memory kernel $\mathcal{K}(t, s) \equiv \alpha^{2} \mathcal{P} \mathcal{L}(t) \mathcal{G}(t, s) \mathcal{Q} \mathcal{L}(s) \mathcal{P}$. Under weak coupling it can be simplified by expanding the Green's function and truncating the expression at order $\alpha^{2}$, which can be done by substituting $\mathcal{G}(t, s)=1+O(\alpha)$, resulting in the equation

$$
\frac{\partial}{\partial t} \mathcal{P} \rho(t)=\alpha \mathcal{P} \mathcal{L}(t) \mathcal{P} \rho(t)+\alpha^{2} \int_{t_{0}}^{t} d s \mathcal{P} \mathcal{L}(t) \mathcal{Q} \mathcal{L}(s) \mathcal{P} \rho(s)
$$

This is not the only useful approximation that can be made, more cases are discussed in ref. [14]. The NZ equation has many applications to non-Markovian dynamics since it is exact and has a clear physical interpretation. For example, one possible approximation could be assuming the memory kernel is some tractable function. For this dissertation though, only the weak coupling approximation will be studied.

Finally, using the explicit definition of the projection operator and assuming $\mathcal{L} \mathcal{P} \rho(t)=0$ one arrives at the equation $3^{3}$

$$
\frac{\partial}{\partial t} \rho_{S}(t)=-\alpha^{2} \int_{t_{0}}^{t} d s \operatorname{tr}_{B}\left[H_{I}(t),\left[H_{I}(s), \rho_{S}(s) \otimes \rho_{B}\right]\right]
$$

This equation is also not easy to solve because it is an integro-differential equation. It is possible to show that under weak coupling approximation substituting $\rho_{S}(s) \rightarrow \rho_{S}(t)$ in the integral

\footnotetext{
${ }^{3}$ This is a common assumption which is true, for a thermal environment for example.
} 
generates errors of the same order in the interaction, but to do so is not trivial(see ref. [14], time convolutionless master equation). Instead this substitution is sometimes called the Markov approximation, even though it does not lead to a Markovian equation either, nor is it actually an approximation. It does result in a local in time equation, which is viable to solve, known as the Redfield equation

$$
\frac{\partial}{\partial t} \rho_{S}(t)=-i \alpha \operatorname{tr}_{B}\left[H_{I}(t), \rho_{S}(t) \otimes \rho_{B}\right]-\alpha^{2} \int_{t_{0}}^{t} d s \operatorname{tr}_{B}\left[H_{I}(t),\left[H_{I}(s), \rho_{S}(t) \otimes \rho_{B}\right]\right]
$$

These weak coupling equations have the downside that if the approximations are not true the resulting map can be negative, meaning the resulting density matrix could have negative eigenvalues. This equation will be applied to a physical problem in the next section.

\subsubsection{Physically motivated microscopic derivation}

In this section we derive the master equation for an atom inside an optical cavity. Cavity environments are a way experimentalists control how the electromagnetic field behaves when coupling to a system. In particular it has a frequency $\omega_{c}$ and only allows dynamics with frequencies near resonance, allowing the atom to be approximated to have only a few levels. The interaction of an atom to the electromagnetic field is described by [18]

$$
H=H_{A}+H_{E}-e \mathbf{r} \cdot \mathbf{E}
$$

where $H_{A}\left(H_{E}\right)$ is the atom's (field's) Hamiltonian. The field Hamiltonian is given by a collection of harmonic oscilators

$$
H_{E}=\sum_{\mathbf{k}} v_{\mathbf{k}}\left(a_{\mathbf{k}}^{\dagger} a_{\mathbf{k}}+\frac{1}{2}\right)
$$

and the field interacts with the atom through its displacement operator

$$
\mathbf{E}=\sum_{\mathbf{k}} \hat{\epsilon}_{\mathbf{k}} \mathscr{E}_{\mathbf{k}}\left(a_{\mathbf{k}}+a_{\mathbf{k}}^{\dagger}\right)
$$

where $\hat{\epsilon}_{\mathbf{k}} \mathscr{E}_{\mathbf{k}}$ are the interaction for different polarizations. The dipole operator can be decomposed in the atom's eigenbases with the identity $\sum_{i}\left|i X_{i}\right|=\mathbb{I}$

$$
e \mathbf{r}=\sum_{i, j} e|i\rangle\langle i|\mathbf{r}| j\rangle\langle j| \equiv \sum_{i, j} \gamma_{i j} \sigma_{i j}
$$


where $\gamma_{i j}=\langle i|\mathbf{r}| j\rangle$ and $\sigma_{i j}=|i\rangle j \mid$, resulting in the Hamiltonian

$$
H=\sum_{\mathbf{k}} v_{k} a_{\mathbf{k}}^{\dagger} a_{\mathbf{k}}+\sum_{i} E_{l} \sigma_{i i}+\sum_{i J} \sum_{\mathbf{k}} g_{\mathbf{k}}^{i j} \sigma_{i j}\left(a_{\mathbf{k}}+a_{\mathbf{k}}^{\dagger}\right)
$$

with the coupling $g_{\mathbf{k}}^{i j}=-\gamma_{i j} \hat{\epsilon}_{\mathbf{k}} \mathscr{E}_{\mathbf{k}}$.

Starting with eq. 2.18 , leads to

$$
\dot{\rho}(t)=-\operatorname{tr}_{E}\left\{\int_{0}^{t} \mathrm{~d} t^{\prime}\left[H_{I}(t),\left[H_{I}\left(t^{\prime}\right), \rho(t) \otimes \rho_{t h}\right]\right]\right\},
$$

where $\rho_{t h}$ is the state of the electromagnetic field (environment), which is henceforth taken to be a thermal state at a certain temperature $T$. Changing variables in eq 2.24 from $t^{\prime}$ to $t-t^{\prime}$ results in

$$
\dot{\rho}(t)=-\operatorname{tr}_{E}\left\{\int_{0}^{t} \mathrm{~d} t^{\prime}\left[H_{I}(t),\left[H_{I}\left(t-t^{\prime}\right), \rho(t) \otimes \rho_{t h}\right]\right]\right\}
$$

and expanding the commutators,

$$
\dot{\rho}(t)=-\operatorname{tr}_{E}\left\{\int_{0}^{t} \mathrm{~d} t^{\prime}\left(H_{I}(t) H_{I}\left(t-t^{\prime}\right) \rho(t) \otimes \rho_{t h}-H_{I}\left(t-t^{\prime}\right) \rho(t) \otimes \rho_{t h} H_{I}(t)\right)+\text { h.c. }\right\} .
$$

This equation is in the interaction picture, so it is convenient to decompose the interaction in eigenoperators defined in the Schrödinger picture by the commutation relation

$$
\left[H_{0}, S(\omega)\right]=\omega S(\omega) \Longrightarrow S_{I}(\omega, t)=\exp \{i \omega t\} S(\omega)
$$

which implies $S(\omega)=S(-\omega)^{\dagger}$. In particular, for the proposed physical system the interaction is already decomposed in eigenoperators with $\omega=\delta(-\delta)$ being the eigenvalue for the eigenoperator $|e\rangle i|(|i\rangle e \mid)$.

For finite systems the eigenoperators are very easy to find. First note that the eigenoperators and their eigenvalues will be given by $\left|\lambda_{i}\right\rangle\left\langle\lambda_{j}\right|$, with $\omega_{i j}=\lambda_{i}-\lambda_{k}$. To obtain an operator decomposition just insert two identities as

$$
A=\sum_{i, k}\left|\lambda_{i} X \lambda_{i}\right| A\left|\lambda_{k} X \lambda_{k}\right|=\sum_{i, k}\left(\left\langle\lambda_{i}|A| \lambda_{k}\right\rangle\right)\left|\lambda_{i} \backslash \lambda_{j}\right|
$$


though in the case proposed of an atom in a cavity the interaction is already written in eigenopperators.

Due to the property $S(\omega)=S(-\omega)^{\dagger}$ and the fact that the sum runs over all values of $\omega$ the decomposition is made such that one of the interactions is conjugate transposed and the other is not. This has two advantages: it makes the secular approximation a lot more convenient 4 it makes obtaining a Lindblad equation easier. In particular the polarization of light will be ignored, as the two channels (one for each polarization) simply do not couple and can be added later. Defining $E(t)=\sum_{k}\left(\gamma_{k}^{*} a_{k}+\gamma_{k} a_{k}^{\dagger}\right)$ we then get

$$
\begin{array}{r}
\dot{\rho}(t)=-\operatorname{tr}_{E}\left\{\sum _ { \omega , \omega ^ { \prime } } \int _ { 0 } ^ { t } \mathrm { d } t ^ { \prime } \left(S^{\dagger}\left(\omega^{\prime}(t)\right) S\left(\omega\left(t-t^{\prime}\right)\right) \rho \otimes E^{\dagger}(t) E\left(t-t^{\prime}\right) \rho_{t h}+\right.\right. \\
\left.\left.-S\left(\omega\left(t-t^{\prime}\right)\right) \rho S^{\dagger}\left(\omega^{\prime}(t)\right) \otimes E\left(t-t^{\prime}\right) \rho_{t h} E^{\dagger}(t)\right)+ \text { h.c. }\right\},
\end{array}
$$

using the time dependence of the eigenoperators and joining the terms which belong to each Hilbert space from the system and bath,

$$
\begin{array}{r}
\dot{\rho}(t)=\sum_{\omega, \omega^{\prime}} \int_{0}^{t} \mathrm{~d} t^{\prime} \exp \left\{i\left(\omega^{\prime}-\omega\right) t\right\} \exp \left\{i \omega\left(t^{\prime}\right)\right\}\left(S^{\dagger}\left(\omega^{\prime}\right) \rho S(\omega) \operatorname{tr}\left\{E\left(t-t^{\prime}\right) \rho_{t h} E^{\dagger}(t)\right\}+\right. \\
\left.-S^{\dagger}\left(\omega^{\prime}\right) S(\omega) \rho \operatorname{tr}\left\{E^{\dagger}(t) E\left(t-t^{\prime}\right) \rho_{t h}\right\}\right)+ \text { h.c. }
\end{array}
$$

results in

$$
\dot{\rho}(t)=\sum_{\omega, \omega^{\prime}} \Gamma(t) \exp \left\{i\left(\omega^{\prime}-\omega\right) t\right\}\left(S\left(\omega^{\prime}\right) \rho S^{\dagger}(\omega)-S^{\dagger}(\omega) S\left(\omega^{\prime}\right) \rho\right)+\text { h.c. }
$$

where

$$
\Gamma(t)=\int_{0}^{t} \mathrm{~d} t^{\prime} \exp \left\{i \omega t^{\prime}\right\} \operatorname{tr}\left\{E^{\dagger}(t) E\left(t-t^{\prime}\right) \rho_{t h}\right\} .
$$

At this point it is necessary to state what the bath looks like in order to calculate the trace in the last expression. If the bath is at zero temperature the trace becomes

\footnotetext{
${ }^{4}$ This approximation will be discussed in more detail in the last chapter
} 


$$
\operatorname{tr}\left\{E^{\dagger}(t) E\left(t-t^{\prime}\right) \rho_{t h}\right\}=\sum_{k, k^{\prime}} \exp \left\{-i \omega_{k} t\right\} \exp \left\{i \omega_{k}^{\prime}\left(t-t^{\prime}\right)\right\} \gamma_{k} \gamma_{k^{\prime}}^{*}\left\langle a_{k} a_{k^{\prime}}^{\dagger}\right\rangle=\int_{0}^{\infty} \mathrm{d} v J(v) \exp \left\{-i v t^{\prime}\right\}
$$

To get a simpler equation one can perform the secular approximation, which assumes that cross terms between $\omega$ 's oscillate fast and can be smoothed out, to get the equation:

$$
\dot{\rho}(t)=\sum_{\omega} \Gamma(t)\left(S(\omega) \rho S^{\dagger}(\omega)-S^{\dagger}(\omega) S(\omega) \rho\right)+\text { h.c. }
$$

One can further simplify this equation by separating $\Gamma$ in real $\gamma$ and imaginary $\lambda$ terms, resulting in the equation

$$
\frac{d}{d t} \rho(t)=-i\left[H_{L S}, \rho(t)\right]+\mathcal{D}(\rho(t), S(\omega)),
$$

where

$$
H_{L S}=\sum_{\omega} \lambda(t) S^{\dagger}(\omega) S(\omega)
$$

and

$$
\mathcal{D}(\rho, S(\omega))=\sum_{\omega} \gamma(t)\left(S(\omega) \rho S^{\dagger}(\omega)-\frac{1}{2}\left\{S^{\dagger}(\omega) S(\omega), \rho\right\}\right) .
$$

An imperfect cavity can be defined by a spectral density $J$ as a sharp Lorentzian, which allows $\Gamma$ to be evaluated analytically. It also allows one extra approximation, that only nearresonant terms contribute to the dynamics, which leaves only two terms, one for each channel. A Lorentzian has two parameters

$$
J(v)=\frac{\alpha^{2}}{\pi} \frac{\Gamma / 2}{\left(v-\omega_{c}\right)^{2}+(\Gamma / 2)^{2}}
$$

a resonant frequency $\omega_{c}$ and its sharpness $\Gamma$ ( $\alpha$ is just the bath's coupling constant). If $\Gamma<<$ $\omega_{c}$ then the integral in eq. 2.33 can be taken from $-\infty$ to $\infty$, which is unphysical because it considers negative frequency modes, but mathematically sound since the spectral density is small in the region where the frequency is negative. This in turn results in

$$
\Gamma(t)=\int_{0}^{t} \mathrm{~d} t^{\prime} \int_{-\infty}^{\infty} \mathrm{d} v J(v) \exp \left\{-i(v-\omega) t^{\prime}\right\}=\int_{0}^{t} \mathrm{~d} t^{\prime} \int_{-\infty}^{\infty} \mathrm{d} v \frac{\alpha^{2}}{\pi} \frac{\Gamma / 2}{\left(v-\left(\omega_{c}-\omega\right)\right)^{2}+(\Gamma / 2)^{2}} \exp \left\{-i v t^{\prime}\right\}
$$


The integral in $v$ is just a Fourier transform of a Lorentzian, and is given by

$$
\mathcal{F}_{v}\left[\frac{1}{\pi} \frac{\Gamma / 2}{\left(v-\omega_{0}\right)^{2}+(\Gamma / 2)}\right](s)=\exp \left\{-2 \pi \mathrm{i} s \omega_{0}-\Gamma \pi|s|\right\}
$$

and the time integral can also be evaluated analytically. Instead of including the results a brief description will be given. Both real and imaginary parts of $\Gamma(t)$ are a combination of an oscillating sine term(with phases) with frequency $\omega-\omega_{c}$ multiplied by a decreasing exponential. They both also have a constant term. This indicates that for long periods of time both terms become constants, which is known as the Markovian limit. In figure 2.1 we present the typical behavior of the functions $\gamma(t)$ and $\lambda(t)$.
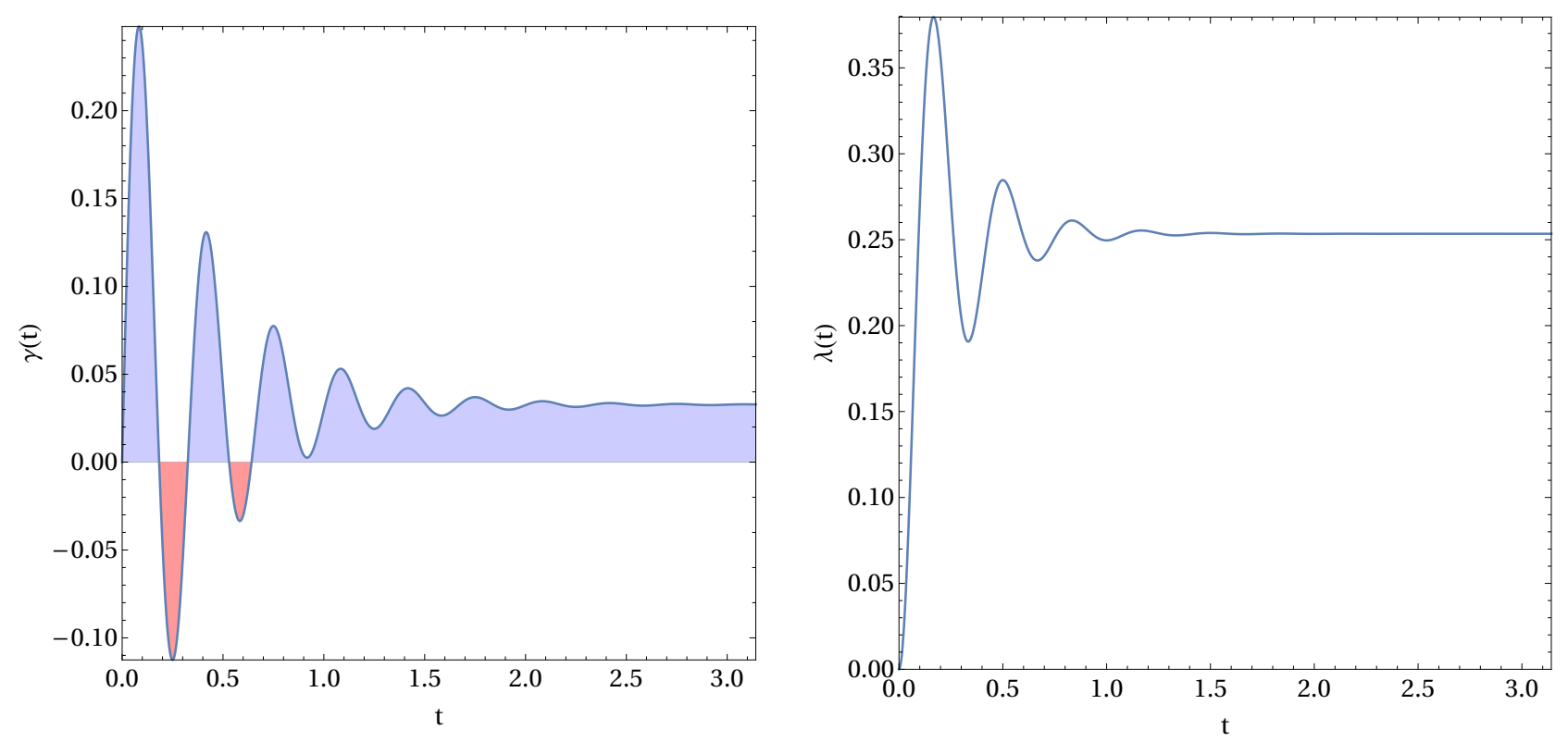

Figure 2.1: Real and imaginary part of $\Gamma(t)$ as in eq. 2.39, with arbitrary parameters, showing both their convergence and the negativity of the real part, which implies non-Markovianity.

$\lambda$ is always positive, and there is not anything interesting in its behavior. $\gamma$ is sometimes negative. This negativity has major implications in the physics involved. It can be shown that equations in the form of eq. 2.35 generate Markovian (divisible) maps if $\gamma$ is always positive. When it is negative the system can recover coherence lost to the bath, and the evolution is non-Markovian.

Finally, by setting a resonant mode (with the same frequency as the channel) to a coherent 
state a laser Hamiltonian is obtained from the term that was ignored in eq. 2.24

$$
H_{L}=\left(\alpha_{L} S(\omega)+\alpha_{L}^{*} S^{\dagger}(\omega)\right)
$$

where the constant $\alpha_{L}$ depends on the displacement of the coherent state and constants related to the coupling to the mode. 


\section{Chapter 3}

\section{Holonomic quantum computation}

In this chapter a description of holonomies and how they apply to quantum computation will be made without much reference to the precise mathematical definition of holonomies in differential geometry, for that see ref. [19]. Instead a heuristic explanation for why these quantum gates are desirable will be made with time dependent perturbation theory.

Holonomic quantum gates are known to be resilient against classical errors in a set of parameters that define its dynamics, though this might not translate to errors due to a quantum environment. For that reason it is interesting to study how these gates behave under a quantum bath.

In particular, a recent paper [20] has shown that under a dephasing noise it is possible to obtain assistance from the environment, meaning the presence of a quantum environment can improve the implementation of the gate.

In this chapter a one qubit holonomic quantum gate will be studied coupled to a bosonic environment. In particular it will be shown that a damping noise can also result in an environment assisted implementation of the gate.

\subsection{Introduction}

The idea of using properties of quantum mechanics to process information was proposed by Feynman in his seminal paper [21]. About a decade later Shor's algorithm was developed [22], which showed quantum computers had an exponential gain for factorization, a very relevant problem in computation with daily applications especially in cryptography. This was one of the first indications that quantum computation could have significant applications. Quantum 
information has already produced interesting protocols such as quantum cryptography (the idea of using quantum mechanics to distribute an encryption key safely), but still, after so many years we have not reached quantum supremacy, when quantum computers perform calculation which would be impossible (practically speaking) on a classical computer.

In the same spirit as classical computation a discrete, or digital, language is currently used. The idea is to implement a set of $N$ two level systems, and with a finite number of gates $U_{\gamma_{i}(k)}$ be able to arbitrarily approximate a unitary with $\left\|U-\prod_{k=1}^{N} U_{\gamma_{i}(k)}\right\| \leq \epsilon$. It turns out that three types of gates are enough to achieve this task, which is known as universality [23].

Implementing a quantum computer ended up being a very daunting task, especially due to decoherence and noise. Studying noise is a central theme in quantum information, especially because quantum systems are in general a lot harder to screen from the environment compared to classical systems. For that reason a series of protocols were created to tackle this problem, in particular error correction, which involves using more qubits than ideally needed to fix errors.

In this dissertation a type of quantum computation that has some built-in noise protection against classical errors in a set of parameters that define its dynamics, known as holonomic quantum computation, will be studied and described with interactions to a bosonic environment. This is motivated by the expectation that the environment could assist in the implementation of the gate.

\subsection{Holonomic quantum gates}

\subsubsection{Berry's phase and Wilczek-Zee phase}

This subsection is based in parts in Sakurai's book in ref. [24], generalized to include the degenerate case of time dependent perturbation theory.

Suppose a system evolves with a time dependent Hamiltonian. The Schrödinger equation for an eigenstate will be given by

$$
H(t)|n ; t\rangle=E_{n}(t)|n ; t\rangle
$$

For a general state the solution can be decomposed in eigenstates

$$
|\alpha ; t\rangle=\sum_{n} c_{n}(t) e^{i \theta_{n}(t)}|n ; t\rangle
$$


with $\hbar=1$ from now on and

$$
\theta_{n}(t) \equiv-\int_{0}^{t} E_{n}\left(t^{\prime}\right) d t^{\prime}
$$

where the phase given by $\theta_{n}$ is called the dynamical phase, and will be useful later. Inputing this state in Schrödinger's equation results in

$$
\sum_{n} e^{i \theta_{n}(t)}\left[\dot{c}_{n}(t)|n ; t\rangle+c_{n}(t) \frac{\mathrm{d}}{\mathrm{d} t}|n ; t\rangle\right]=0
$$

Assuming that there is no degeneracy, looking at one particular amplitude in the eigenbases gives

$$
\dot{c}_{m}(t)=-c_{m}(t)\langle m ; t|\left[\frac{\mathrm{d}}{\mathrm{d} t}|m ; t\rangle\right]-\sum_{n} c_{n}(t) e^{i\left(\theta_{n}-\theta_{m}\right)} \frac{\langle m ; t|\dot{H}| n ; t\rangle}{E_{n}-E_{m}} .
$$

If the Hamiltonian evolves very slowly in time compared with the differences between energy levels, then the second term can be neglected, which results in the solution

$$
\begin{aligned}
& c_{n}(t)=e^{i \gamma_{n}(t)} c_{n}(0), \\
& \gamma_{n}(t) \equiv i \int_{0}^{t}\left\langle n ; t^{\prime}\right|\left[\frac{\mathrm{d}}{\mathrm{d} t^{\prime}}\left|n ; t^{\prime}\right\rangle\right] d t^{\prime} .
\end{aligned}
$$

This extra phase is known as Berry's phase [25]. This phase took many years to be considered relevant, in particular because it was not clear if it could be measured. It was later discovered that it plays a major role in cyclic time evolutions.

If the Hamiltonian's time dependence can be described by a vector of parameters $\mathbf{R}(t)$ which belong to a smooth manifold, then the integrand in eq. 3.5, using the chain rule, can be rewritten as

$$
i\langle n ; \mathbf{R}|\left[\frac{\mathrm{d}}{\mathrm{d} t}|n ; \mathbf{R}\rangle\right]=i\left\langle n ; \mathbf{R}\left|\nabla_{\mathbf{R}}\right| n ; \mathbf{R}\right\rangle \cdot \frac{\mathrm{d} \mathbf{R}}{\mathrm{d} t} \equiv \mathbf{A}_{n}(\mathbf{R}) \cdot \frac{\mathrm{d} \mathbf{R}}{\mathrm{d} t}
$$

This allows the integral to be evaluated along a curve in parameter space by changing variables in the integral in eq. 3.6 .

$$
\gamma_{n}(T)=i \int_{0}^{T} \mathbf{A}_{n}(\mathbf{R}) \cdot \frac{\mathrm{d} \mathbf{R}}{d t} d t=\int_{C} \mathbf{A}_{n}(\mathbf{R}) \cdot \mathrm{d} \mathbf{R} .
$$

If the time evolution is cyclic, with a period $T$ (meaning $\mathbf{R}(0)=\mathbf{R}(T)$ ), then using Stokes' theorem results in

$$
\gamma_{n}(T)=\oint_{C} \mathbf{A}_{n}(\mathbf{R}) \cdot \mathrm{d} \mathbf{R}=\int\left[\nabla_{\mathbf{R}} \times \mathbf{A}_{n}(\mathbf{R})\right] \cdot \mathrm{d} \mathbf{a},
$$

where da is a vector of measures for each parameter. 
The fact that the phase only depends on the total flux $\nabla_{\mathbf{R}} \times \mathbf{A}_{n}(\mathbf{R})$ indicates that this phase is robust against random errors in $\mathbf{R}$ (for an illustration of this, see fig. 3.1 with an example where the parameter manifold is a unit sphere).
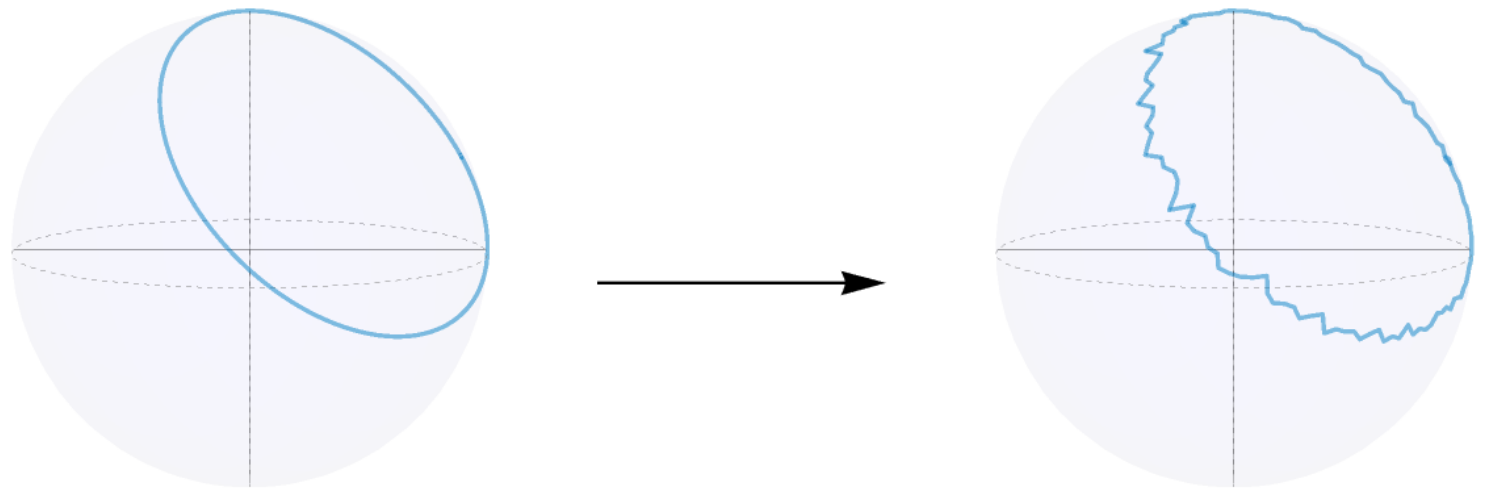

Figure 3.1: Illustrative example of how random noise in the parameter space will not change the phase significantly, since it only depends on the flux through the curve.

This vector field $\mathbf{A}$ is analogous to a vector potential when considering the transformation

$$
\begin{aligned}
|n ; t\rangle & \longrightarrow e^{i \delta(\mathbf{R})}|n ; t\rangle \\
\mathbf{A}_{n}(\mathbf{R}) & \longrightarrow \mathbf{A}_{n}(\mathbf{R})-\nabla_{\mathbf{R}} \delta(\mathbf{R})
\end{aligned}
$$

which in turn implies the curl of $\mathbf{A}$ is analogous to a magnetic field.

Assuming the initial state belongs to a degenerate subspace, using the previous results one can conclude that the system will stay in this as seen in eq. 3.6. Then the time evolution of the coefficient will have an extra sum

$$
\dot{c}_{m}(t)=-\sum_{n} c_{n}(t)\langle m ; t|\left[\frac{\mathrm{d}}{\mathrm{d} t}|n ; t\rangle\right] \equiv i M_{m n}(t) c_{n}(t) .
$$

In the last term Einstein sum notation was used. This will be the case for the whole chapter.

The solution for the vector of coefficients $c_{m}$ will now be a matrix exponential

$$
c_{m}(t)=T \exp \left\{i \int_{0}^{t} \mathrm{~d} t M_{m n}(t)\right\} c_{n}(0)
$$


where $T$ is the time ordering operator. It is also possible, using the chain rule again, to define a matrix-valued vectors potential analogous to eq. 3.7

$$
\mathbf{A}_{m n}(\mathbf{R})=\left\langle m ; \mathbf{R}\left|\nabla_{\mathbf{R}}\right| n ; \mathbf{R}\right\rangle .
$$

This implies that a unitary is implemented in the subspace, given by

$$
U(T)=P \exp \left\{i \int_{C} \mathbf{A}_{m n}(\mathbf{R}) \cdot \mathrm{d} \mathbf{R}\right\}
$$

where $P$ is the path ordering. This unitary is also robust to noise if the curve described by $\mathbf{R}$ is closed for the same reason as in the Berry's phase case. The "phase" in the degenerate case is known as Wilzcek-Zee phase [26].

In analogy to the gauge transformation in eq. 3.10 , instead of a time dependent phase one can apply a time dependent unitary to the degenerate subspace, which in turn implies the matrix A transforms as

$$
\begin{aligned}
\left|n^{\prime} ; t\right\rangle & =\Omega(t)|n ; t\rangle \\
\mathbf{A}^{\prime}(t) & =\dot{\Omega} \Omega^{-1}+\Omega \mathbf{A} \Omega^{-1}
\end{aligned}
$$

which is how gauge fields tranform. In particular, the holonomies are analogous to Wilson loops, which are gauge invariant [27].

\section{Adiabatic holonomic quantum gates}

The protocol of generating a geometric phase in a cyclic evolution is known as a holonomy, in particular in the degenerate case a non-Abelian holonomy, after all the resulting unitary matrices might not commute. In this section a one-qubit quantum gate using only holonomies will be described.

In order to obtain a universal quantum computer a set of two one-qubit gates and one twoqubit gate is necessary. In this project only one-qubit gates will be described, though it is also possible to obtain a two-qubit gate using only non-Abelian holonomies as well.

We consider a tripod system (see fig. 3.2) described by the Hamiltonian [28]

$$
H=\left[|e\rangle\left(\Omega_{0}\langle 0|+\Omega_{1}\langle 1|+\Omega_{a}\langle a|\right)+\text { h.c. }\right] .
$$


This Hamiltonian has a doubly degenerate dark state (meaning they do not contain the excited state, $|e\rangle)$ subspace where the non-Abelian holonomy will be implemented.

Assuming the degenerate subspace is spanned by the vectors $\left|D_{1}\right\rangle$ and $\left|D_{2}\right\rangle, \mathbf{A}$ as in eq. 3.13 will be given by

$$
\mathbf{A}_{i j}=\left\langle D_{i}\left|\nabla_{\mathbf{R}}\right| D_{j}\right\rangle
$$

and the resulting non-Abelian holonomy for a closed path

$$
U(C)=P \exp \left\{i \oint_{C} \mathbf{A}(\mathbf{R}) \cdot \mathrm{d} \mathbf{R}\right\}
$$

where $P$ is the path ordering operator.

As an example, if we parametrize $\Omega_{0}=\Omega \sin \theta \cos \phi, \Omega_{1}=\Omega \sin \theta \sin \phi$ and $\Omega_{a}=\Omega \cos \theta$, the degenerate subspace will be spanned by $\left|D_{1}\right\rangle=\cos \theta(\cos \phi|0\rangle+\sin \phi|1\rangle)-\sin \theta|a\rangle$ and $\left|D_{2}\right\rangle=\cos \phi|1\rangle-\sin \phi|0\rangle$, and each component of the vector $\mathbf{A}$ will be given by

$$
A_{\theta}=\left(\begin{array}{ll}
0 & 0 \\
0 & 0
\end{array}\right) \quad \text { and } \quad A_{\phi}=\sigma_{y} \cos \theta
$$

resulting in the holonomy

$$
U(C)=\exp \left\{i \sigma_{y} \oint_{C} \cos \theta \mathrm{d} \phi\right\}
$$

where the path ordering is no longer needed, and the integral is simply the solid angle generated by the path of $\theta$ and $\phi$. Suppose the solid angle is $\pi / 4$, then the implemented unitary will be

$$
U(C)=\exp \left\{i \pi \sigma_{y} / 4\right\}=\frac{1}{\sqrt{2}}\left(\begin{array}{cc}
1 & 1 \\
-1 & 1
\end{array}\right)=\mathrm{H} \cdot X
$$

where $\mathrm{H}$ is the Hadamard gate and $X$ is a bit flip.

To make sure the gate is implemented in the computational bases the parameters at the beginning of the path can be chosen in such a way that the dark states are initially $|0\rangle$ and $|1\rangle$. One can show, with a different choice of parameters, that another gate that, joined with this one, generate a universal one-qubit gate.

This approach can also be generalized by using a $N+2$ level system, which in turn has a $N$ dimensional degenerate subspace where a non-Abelian holonomy can be implemented [29]. This could, for instance, be used for a two-qubit gate. 

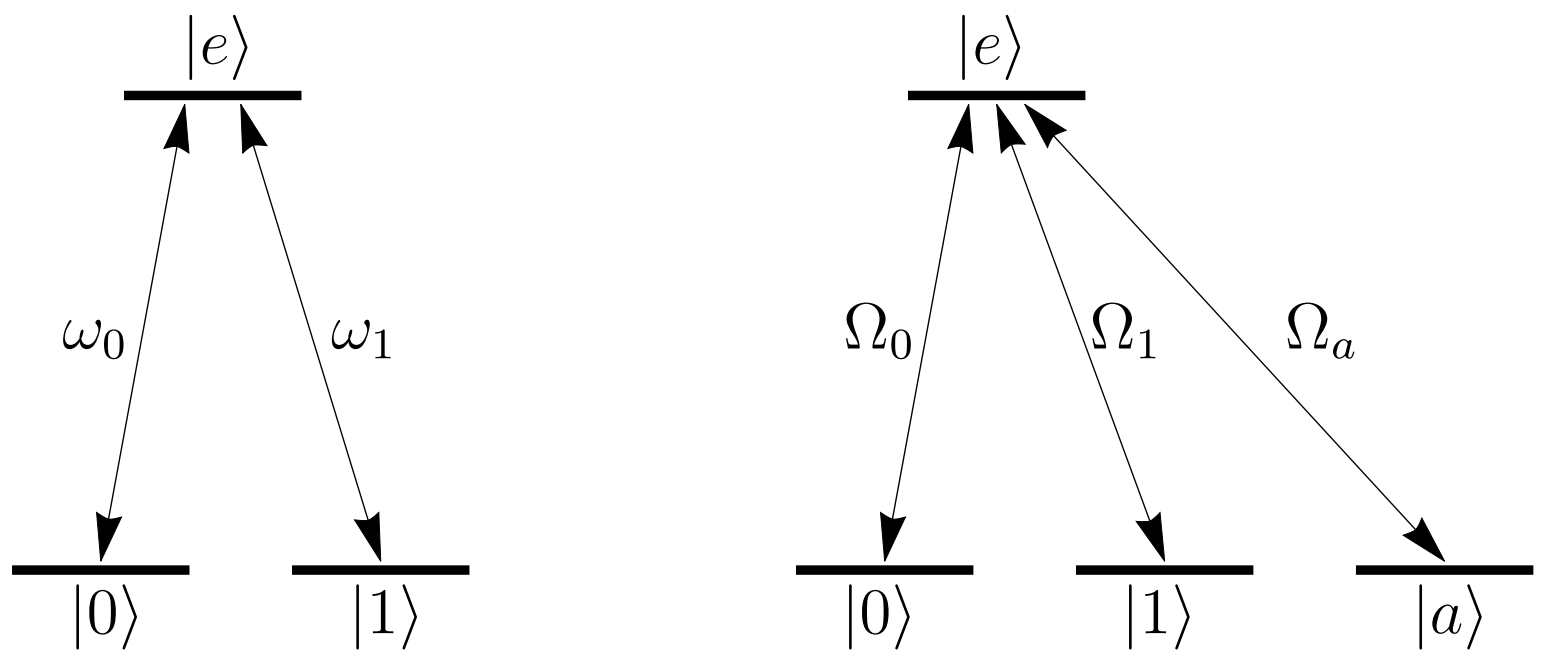

Figure 3.2: Energy level and couplings setup required for non-adiabatic and adiabatic (respectively a lambda system and a tripod system) implementations of a one-qubit holonomic quantum gate [28].

\subsubsection{Non-adiabatic holonomic quantum gates}

Although holonomic quantum gates are robust against some errors, being adiabatic is a disadvantage if one is interested in implementing a quantum gate since adiabaticity restricts how fast it can be implemented and also exposes the system to the environment for, in some sense, a long time. For this reason the gate described above is not easy to implement experimentally, though it has been done [30]. More recently a non-adiabatic version, using only three energy levels was proposed, only requiring one dark state.

\section{Non-adiabatic holonomies}

The geometric and dynamical contribution to the dynamics can be separated by writing the time evolution operator as

$$
U(t)=T \exp \left\{\int_{0}^{t} \mathrm{i}(A-K) \mathrm{d} t\right\}
$$

where the matrices $A$ and $K$ are given by

$$
A_{a b}=i\left\langle\psi_{a}\left|\frac{\mathrm{d}}{\mathrm{d} t}\right| \psi_{b}\right\rangle \quad \text { and } \quad K_{a b}=\left\langle\psi_{a}|H| \psi_{b}\right\rangle
$$

and $\left|\psi_{a}(t)\right\rangle$ is an orthonormal bases. 
The interpretation of $A$ as the geometrical contribution to the time evolution comes from the fact that it does not depend on the Hamiltonian, but only in geometrical properties of the Hilbert space [31]. With this in mind a non-adiabatic holonomic quantum gate can be implemented by finding a process and a Hamiltonian with $K_{a b}=0$ for all $t$, then, with a cyclic evolution, a similar robustness to error as in the adiabatic case should be expected.

It is interesting to note that this matrix also has the same gauge structure as the adiabatic case, with a unitary $\Omega$ taking one bases to the other, the geometric and dynamical contributions transform as

$$
A \rightarrow \mathrm{i} \Omega^{\dagger} \hat{\Omega}+\Omega^{\dagger} A \Omega \text { and } K \rightarrow \Omega^{\dagger} K \Omega
$$

\section{Zero detuning implementation}

One possible way to implement a non-adiabatic quantum gate is to start with the Hamiltonian [32]

$$
H(t)=\Omega(t)\left(\omega_{0}|e\rangle\left\langle 0\left|+\omega_{1}\right| e\right\rangle\langle 1|+\text { h.c. }\right)
$$

with $\left|\omega_{0}\right|^{2}+\left|\omega_{1}\right|^{2}=1$, which has a dark state $|d\rangle=-\omega_{1}|0\rangle+\omega_{0}|1\rangle$ and a bright one $|b\rangle=$ $\omega_{0}^{*}|0\rangle+\omega_{1}^{*}|1\rangle$. The Hamiltonian can then be rewritten as

$$
H(t)=\Omega(t)(|e X b|+h . c .) \rightarrow \Omega(t) \tau_{x}
$$

which is simply a Rabi oscillation of a qubit spanned by $\{|e\rangle,|b\rangle\}$, with $\tau_{x}$ being the Pauli operator in the $e-b$ subspace. In this bases the time evolution operator is given by

$$
U(t)=\exp \left\{-i \tau_{x} \int_{0}^{t} \mathrm{~d} t \Omega(t)\right\}
$$

By choosing $\int_{0}^{t} \mathrm{~d} t \Omega(t)=\pi$ the resulting gates simply does the operation $|b\rangle \rightarrow-|b\rangle,|e\rangle \rightarrow$ $-|e\rangle$ and $|d\rangle \rightarrow|d\rangle$. In order to figure out what unitary will be implemented in the computational bases one can interpret this operation as a $\sigma_{z}$ applied to the bases $|d\rangle$ and $|b\rangle$. Using the parametrization $\omega_{0}=\sin (\theta / 2) e^{i \phi}, \omega_{1}=-\cos (\theta / 2)$, which in turn defines a unit vector $\mathbf{n}=\{\sin \theta \sin \phi, \sin \theta \cos \phi, \cos \theta\}$ one can then define a change of bases matrix

$$
\exp \left\{i \mathbf{n} . \vec{\sigma} \frac{\pi}{2}\right\}\left(\begin{array}{l}
|1\rangle \\
|0\rangle
\end{array}\right)=\left(\begin{array}{l}
|b\rangle \\
|d\rangle
\end{array}\right)
$$


which implies

$$
\sigma_{z} \rightarrow \exp \left\{-i \mathbf{n} \cdot \vec{\sigma} \frac{\pi}{2}\right\} \cdot \sigma_{z} \cdot \exp \left\{i \mathbf{n} \cdot \vec{\sigma} \frac{\pi}{2}\right\}=\mathbf{n} \cdot \vec{\sigma}
$$

in the computational bases. This can be seen as a purely geometric evolution since the dynamical phase, given by $\left\langle\psi_{k}(t)|H(t)| \psi_{l}(t)\right\rangle=\langle k|H(t)| l\rangle$ (because $[U(t), H]=0$, for all $t$ ) is zero throughout the evolution. The unitary holonomy in eq. 3.29 is traceless, but it is possible to implement a general unitary by using two gates, with $U(C)=U\left(C_{\mathrm{m}}\right) U\left(C_{\mathrm{n}}\right)=\mathbf{n} \cdot \mathbf{m}-\mathrm{i} \sigma \cdot(\mathbf{n} \times \mathbf{m})$.

\section{Single-shot realization}

Performing two gates can be a problem since the system will be exposed to the environment for twice the time. For that reason a single-shot implementation would be interesting. It turns out to be possible to implement a geometric gate as long as one has a controllable detuning, and it has even been experimentally implemented in Xmon qutrits [33]. The single shot implementation is done with the Hamiltonian

$$
\mathcal{H}(t)=\Omega(t) \sin \alpha|e\rangle\langle e|+\frac{1}{2} \Omega(t) \cos \alpha(|e\rangle\langle b|+| b\rangle\langle e|)
$$

with

$$
\int_{0}^{\tau} \frac{\Omega(t)}{2} d t=\pi
$$

which generate a time evolution operator $U(\tau)=|d\rangle\left\langle d\left|+e^{-i \pi(1+\sin \alpha)}\right| b\right\rangle\left\langle b\left|+e^{-i \pi(1+\sin \alpha)}\right| e\right\rangle\langle e|$, that can be projected in the qubit subspace resulting on the universal single qubit gate $U_{L}(\tau)=$ $e^{-i \gamma n \cdot \sigma / 2}$, with $\gamma=1+\sin \alpha$.

\subsection{Environment assisted implementation}

One of the main problems related to the non-adiabatic holonomic quantum gate described previously is that the qubit couples to an excited state. This means that when noise or parametric errors are added there is a chance that the non-qubit level is occupied in the end of the implementation.

Using the zero temperature environment proposed in the previous chapter, there are decays from the exited state to the qubit subspace, which makes it seems reasonable to believe that it 
could be used to fix errors.

In this section the traceless version of the holonomic quantum gate will be implemented using square pulses with a gaussian noise in the pulse time. This imposes a smaller than 1 fidelity even when uncoupled to the environment.

\subsubsection{Initial states and pulse error}

The gate fidelity will be averaged out on random initial states uniformly distributed on the Bloch sphere. The random unit vector that describes the state can be obtained by generating three normally distributed numbers defining a vector and then normalizing it [34]. A square pulse will be implemented with a runtime of $\pi$ plus a gaussian noise with some arbitrary variance, which will result in a lower than 1 fidelity even when uncoupled to the environment.

\subsubsection{Master equation}

The $X$ gate was arbitrarily chosen for illustration purposes. The master equation in the interaction picture to implement an $X$ gate is

$$
\dot{\rho}=-i\left[H_{L}, \rho\right]-i \lambda(t)\left[H_{L S}, \rho\right]+\gamma(t)(\mathcal{D}(\rho,|0 \backslash e|)+\mathcal{D}(\rho,|1 \backslash e|))
$$

with

$$
H_{L}=\left(\begin{array}{ccc}
0 & \frac{1}{\sqrt{2}} & -\frac{1}{\sqrt{2}} \\
\frac{1}{\sqrt{2}} & 0 & 0 \\
-\frac{1}{\sqrt{2}} & 0 & 0
\end{array}\right)
$$

The Lamb-shift term will be ignored since it can be effectively removed in a rotating frame and tuning the laser properly.

\subsubsection{Markovian limit noise}

In the markovian limit it is assumed that the system's dynamics is so much slower than the bath's that the time integral in eq. 2.39 can be approximated as having a limit in infinite time. This can be shown to generate a time evolution that forms a semi-group, which is the definition of a Markovian evolution in quantum mechanics [14]. Markovianity plays a central role in open quantum systems, in particular because there are theorems that guarantee CPTP in very general and well understood situations. 
The resulting master equation will be

$$
\dot{\rho}=-i\left[H_{L}, \rho\right]+\gamma(\infty)\left(\mathcal{D}\left(\rho,\left|0 X_{e}\right|\right)+\mathcal{D}(\rho,|1 \times e|)\right)
$$

In fig. 3.3 the average fidelity $\mathcal{F}$ is plotted as a function to the coupling to the environment $\gamma(\infty) \equiv \gamma$. In this case the environment will always harm the fidelity.

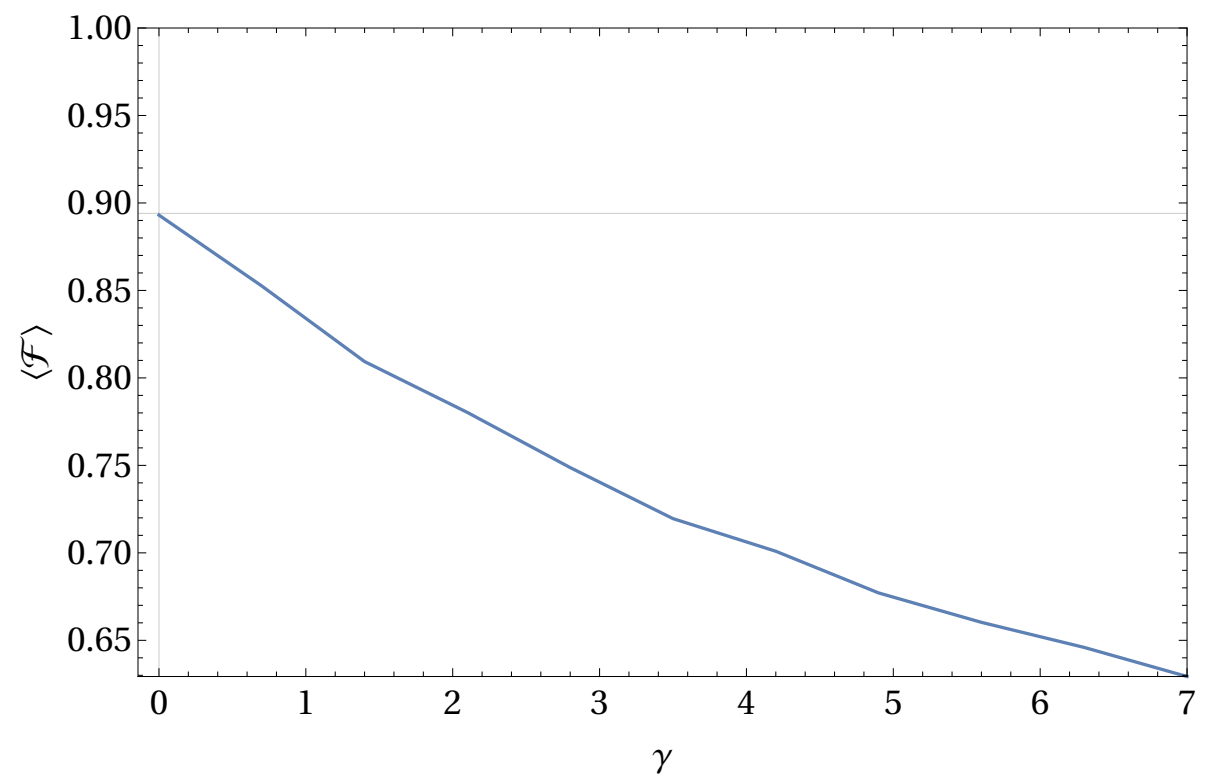

Figure 3.3: Average fidelities for an $X$ gate coupled to a markovian environment

\subsubsection{Including a relaxation time}

A possible way of using the environment to increase the fidelity is to leave the system in the environment for it to be projected in the qubit subspace for a relaxation time ("R") after the implementation of the gate. In a practical implementation this would have drawbacks since there usually are more sources of noises such as dephasing. For that reason it is interesting to look at different finite relaxation times, such as in fig 3.4 


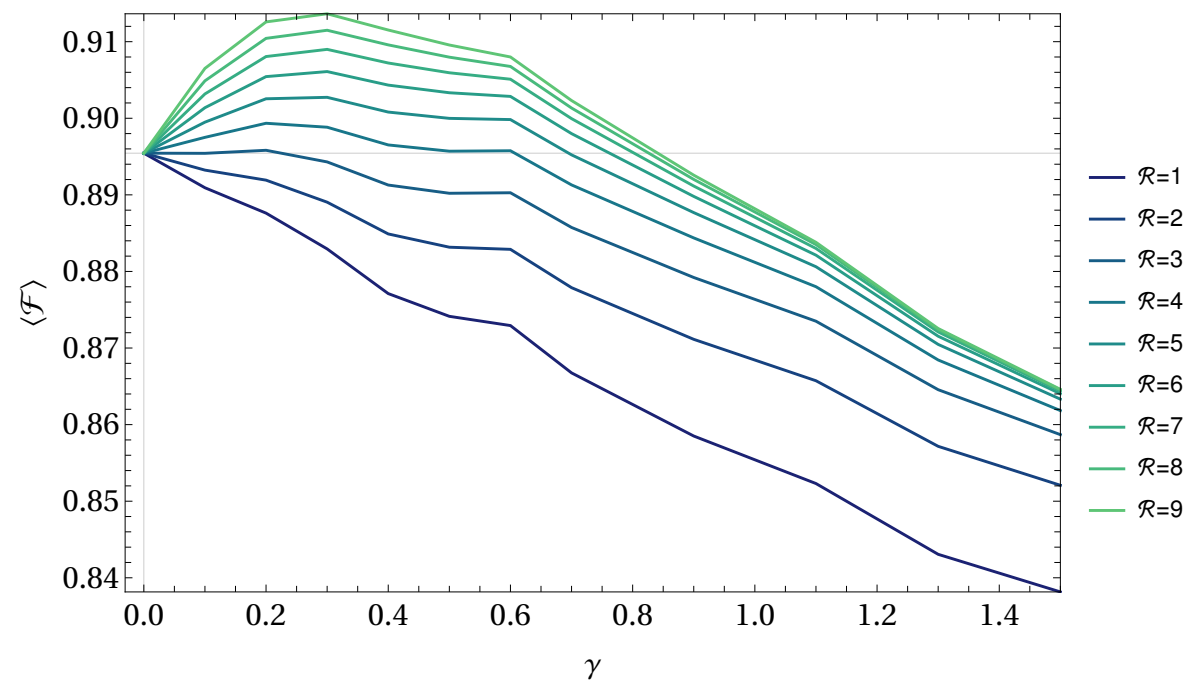

Figure 3.4: Average fidelities for an $X$ gate coupled to a Markovian environment with diferent relaxation times $\mathcal{R}$.

This result is interesting because the gate becomes more accurate with the environment than without it, which is known as environmental assistance. There is a competition between the environment reducing the fidelity during the implementation but also helping it by projecting in the qubit subspace during the relaxation, which generates an optimal coupling strength. If the interaction is very weak then the implementation is optimal, but the necessary relaxation time to allow assistance becomes large. On the other hand if the interaction is too strong then no relaxation time is able to provide assistance since the fidelity during the implementation becomes too low.

\subsection{Future perspectives}

In this chapter, an overview on holonomic quantum computation and open quantum systems was made. It was shown that a Markovian environment could assist in the implementation of a non-adiabatic holonomic quantum gate by addressing a relevant cause of errors due to the population of the exited state.

We believe that this protocol could be further improved if more properties such as nonMarkovianity (negativity of the damping coefficient) are considered (see fig. 3.5), though there are technical problems involving non-Markovianity and weak coupling derivations, such as the lack of strong non-Markovianity, which is the case where there is a back-flow of information from the bath to the system.

Other possible approaches could involve simpler interactions to the environment, such as a 
$|e \times b|$ coupling with the intent to obtain exact solutions, or more abstract models for the bath, such as collisional models.

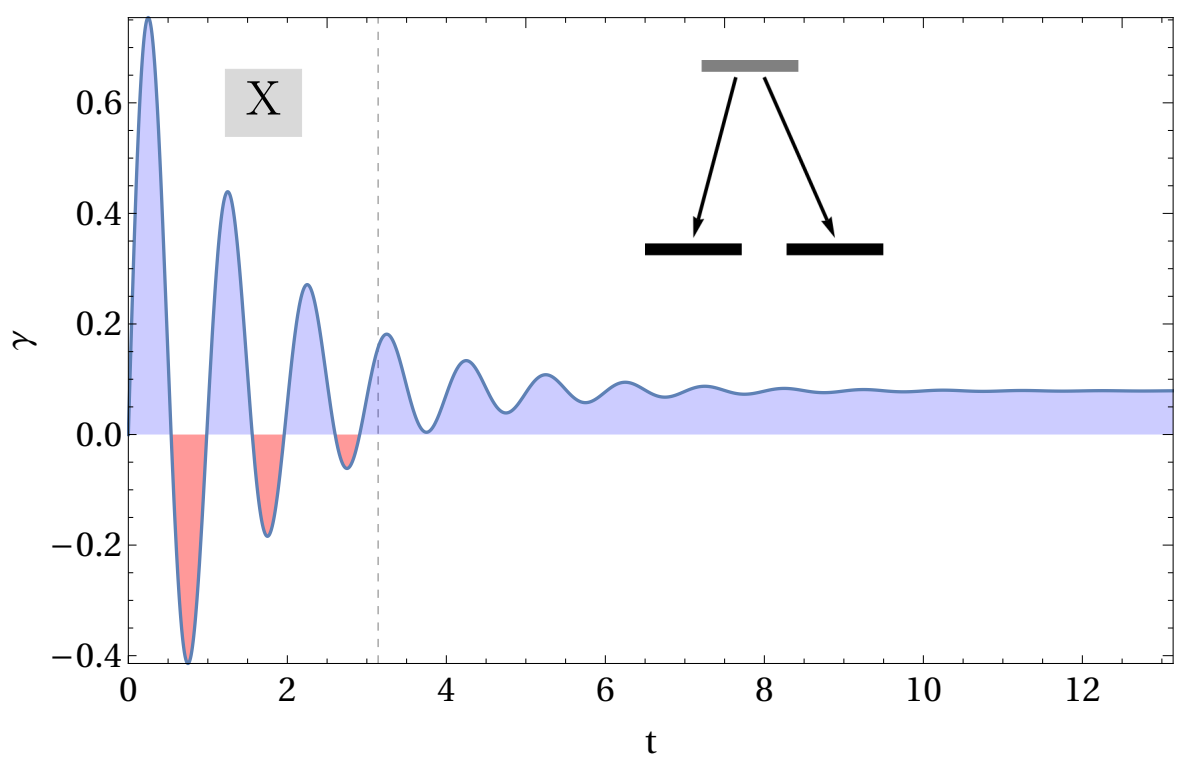

Figure 3.5: Illustration of the protocol, where the $X$ gate in implemented when the evolution is non-Markovian and then left to decay into the qubit subspace in the Markovian regime. 


\section{Chapter 4}

\section{Thermodynamics of Bosonic Gaussian states}

\subsection{Wigner representation and quantum Gaussian states}

This section is based in part on [35].

We consider here a Bosonic system with $L$ modes, characterized by creation and annihilation operators $a_{i}^{\dagger}$ and $a_{i}$. To any density matrix of the system, one can associate the corresponding Wigner function as

$$
W(\boldsymbol{\xi})=\frac{1}{\pi^{2 L}} \int \mathrm{d} \boldsymbol{\lambda} e^{-\sum_{i=1}^{2 L}\left(\lambda_{i} \alpha_{i}^{*}-\lambda_{i}^{*} \alpha_{i}\right)} \operatorname{tr}\left\{\rho e^{\sum_{i=1}^{2 L}\left(\lambda_{i} a_{i}^{\dagger}-\lambda_{i} a_{i}\right)}\right\},
$$

where $\xi^{\mathrm{T}}=\left(\alpha_{1}, \alpha_{1}^{*}, \ldots, \alpha_{L}, \alpha_{L}^{*}\right)$ and where the integration measure is $\mathrm{d} \lambda=\prod_{i=1}^{L} \mathrm{~d} \operatorname{Re}\left(\lambda_{i}\right) \mathrm{d} \operatorname{Im}\left(\lambda_{i}\right) ;$ i.e., over the entire complex plane of each variable $\lambda_{i}$. The Wigner function contains the same amount of information as the density matrix, but represents it in quantum phase space, a $L$ dimensional complex space, where $L$ is the number of modes.

There is a particularly useful set of states, called Gaussian states, which are defined as states with a Gaussian Wigner function. They are particularly relevant in quantum optical systems and include coherent states. They are also appealing to quantum information due to the fact that they can be described and simulated more easily than otherwise, while still having interesting quantum properties such as entanglement. 
The Wigner function for Gaussian states is given by

$$
W(\xi)=\frac{1}{\pi^{L} \sqrt{|\Theta|}} \exp \left\{-\frac{1}{2}(\xi-\mu)^{\dagger} \Theta^{-1}(\xi-\mu)\right\},
$$

where

$$
\Theta_{i j} \equiv \frac{1}{2}\left\langle\left(\left(a_{i}-\left\langle a_{i}\right\rangle\right)\left(a_{j}^{\dagger}-\left\langle a_{j}^{\dagger}\right\rangle\right)+i \leftrightarrow j\right)\right\rangle
$$

is the Covariance Matrix (CM) and $\mu=\left(\mu_{1}, \mu_{1}^{*}, \mu_{2}, \mu_{2}^{*}, \ldots\right)$, with $\mu_{i}=\left\langle a_{i}\right\rangle$.

It is particularly interesting that a Lindblad equation of the form

$$
\dot{\rho}=-\mathrm{i}\left[\sum_{i j}\left(\omega_{i j} a_{i}^{\dagger} a_{j}\right), \rho\right]+\sum_{i j} \gamma_{i j}^{-} \mathrm{D}\left[a_{i}, a_{j}^{\dagger}, \rho\right]+\sum_{i j} \gamma_{i j}^{+} \mathrm{D}\left[a_{i}^{\dagger}, a_{j}, \rho\right]
$$

which has a quadratic Hamiltonian and a linear jump operator (with respect to the creation and annihilation operators, where $\mathbb{D}$ was defined in eq. 2.5p, preserves Gaussianity, in the sense that if the state is initially Gaussian, it will remain so for all future times. 1 . Since Gaussian states are completely characterized by the first and second moments, this means that the evolution under Eq. (4.3) can be solely cast in terms of $\mu$ and $\Theta$. The first moments evolve according to

$$
\frac{d \mu}{d t}=-W \mu
$$

whereas the covariance matrix evolves according to the Lyapunov equation:

$$
\dot{\Theta}=-\left(W \Theta+\Theta W^{\dagger}\right)+\mathcal{D}
$$

where the form of the matrices $W$ and $\mathcal{D}$ will be discussed in more detail in the next chapters.

A very useful property of Gaussian states is the easeness with which one can take partial traces. Since the marginal of a Gaussian state is still Gaussian [35], to obtain the partial trace one simple removes the entries of the $\mathrm{CM}$ related to the variables one wishes to trace out. For

\footnotetext{
${ }^{1}$ This is not the most general quadratic Lindblad equation. It does not include squeezing for example, but it is enough for this dissertation.
} 
instance, if we consider a bipartite system with a state $\rho_{A B}$ characterized by a CM

$$
\Theta_{A B}=\left(\begin{array}{cc}
\Theta_{A} & \Theta_{A B} \\
\Theta_{A B}^{\dagger} & \Theta_{B}
\end{array}\right)
$$

then the $\mathrm{CM}$ of the reduced density matrix $\rho_{A}$ will simply be $\Theta_{A}$.

\subsection{Wigner entropy}

Although it is possible to calculate the von-Neumann entropy for Gaussian states, a much simpler, alternative, definition is to consider

$$
S(W)=-\int \mathrm{d} \boldsymbol{\xi} W(\boldsymbol{\xi}) \ln W(\boldsymbol{\xi})
$$

which, as we now discuss, has many advantages. We call this the Wigner entropy. It was first introduced in [36] and also further explored in [37]. In ref. [36] it was shown that for Gaussian states this entropy satisfies the strong subadditivity inequality

$$
S(A B)+S(B C) \geq S(A B C)-S(B)
$$

which, in general, is a property that holds only for the von Neumann entropy. This alone is a strong case for the Wigner entropy. In addition, one can also show that for Gaussian states this coincides with the Rényi-2 entropy [36], defined as

$$
S_{2}(\rho)=-\ln \operatorname{tr}\left(\rho^{2}\right)
$$

The relation between them is established as follows. The purity of a Gaussian state are given by

$$
\operatorname{tr}\left(\rho^{2}\right)=\frac{1}{2^{L} \sqrt{|\Theta|}}
$$

which implies

$$
S_{2}(\Theta)=\frac{1}{2} \ln |\Theta|+L \ln 2
$$


Similarly, plugging Eq. (4.2) into Eq. (4.6) and carrying out the Gaussian integrals, one finds that

$$
S=\frac{1}{2} \ln |\Theta|+1+\ln \pi
$$

This formula is extremely convenient, as it offers a straightforward route to compute the entropy of Gaussian states. For example, the mutual information

$$
I_{A B}=S_{A}+S_{B}-S_{A B}=\frac{1}{2} \ln \left\{\frac{\left|\Theta_{A}\right|\left|\Theta_{B}\right|}{\left|\Theta_{A B}\right|}\right\},
$$

and the rate of change of the entropy as

$$
\frac{\mathrm{d} S}{\mathrm{~d} t}=\frac{1}{2} \operatorname{tr}\left\{\Theta^{-1} \frac{\mathrm{d} \Theta}{\mathrm{d} t}\right\}
$$

where the property $\log |\Theta|=\operatorname{tr}\{\log \Theta\}$, which can be proven by diagonalizing the matrix and using properties of log, was used.

\subsection{Quantum Fokker-Plank equation}

As we have seen, any quantum state can also be represented by a Wigner function. Equivalently the time evolution of the quantum state, given by a Lindblad equation, can be represented by a differential equation known as a quantum Fokker-Plank equation [4].

To illustrate the idea, we consider here the simplified case of a single Bosonic mode coupled to a thermal Lindblad equation. In this case the master equation is given by

$$
\frac{d \rho}{d t}=-i\left[\omega a^{\dagger} a, \rho\right]+\gamma(\bar{n}+1) \mathbb{D}\left[a, a^{\dagger}, \rho\right]+\gamma \bar{n} \mathrm{D}\left[a^{\dagger}, a, \rho\right]
$$

The Fokker-Planck equation can be calculated in this case using standard correspondence tables [38]:

$$
\begin{aligned}
& a \rho \leftrightarrow\left(\alpha+\frac{1}{2} \frac{\partial}{\partial \alpha^{*}}\right) W\left(\alpha, \alpha^{*}\right) \\
& a^{\dagger} \rho \leftrightarrow\left(\alpha^{*}-\frac{1}{2} \frac{\partial}{\partial \alpha}\right) W\left(\alpha, \alpha^{*}\right) \\
& \rho a \leftrightarrow\left(\alpha-\frac{1}{2} \frac{\partial}{\partial \alpha^{*}}\right) W\left(\alpha, \alpha^{*}\right) \\
& \rho a^{\dagger} \leftrightarrow\left(\alpha^{*}+\frac{1}{2} \frac{\partial}{\partial \alpha}\right) W\left(\alpha, \alpha^{*}\right)
\end{aligned}
$$


By collecting all terms in a generic Lindblad equation we get

$$
\frac{\mathrm{d} W}{\mathrm{~d} t}=-i \omega\left[\frac{\partial}{\partial \alpha^{*}}\left(\alpha^{*} W\right)-\frac{\partial}{\partial \alpha}(\alpha W)\right]+\tilde{D}(W)
$$

where the first terms represent the unitary contribution from the Hamiltonian, and the last term is a dissipative part from the Lindblad terms

$$
\begin{aligned}
& \tilde{D}(W)=\partial \mathcal{J}(W)+\partial^{*} \mathcal{J}^{*}(W) \\
& \mathcal{J}(W)=\frac{\gamma}{2}\left(\alpha W+(\bar{n}+1 / 2) \partial^{*} W\right)
\end{aligned}
$$

Similar results can be obtained with multiple modes, and this will be relevant to define the Wigner entropy production, as we shall do next.

\subsection{Entropy production}

During a thermodynamic process, not all entropy change in the system can be associated with a flow of entropy from the environment. Instead, some entropy may also be irreversibly produced during the process. This entropy production therefore serves to characterize the degree of irreversibility of a given process. It is expected to have the important property that it must be non-negative, and only zero in thermal equilibrium.

In symbols, we can write the variation of the entropy as a function of time as

$$
\frac{\mathrm{d} S}{\mathrm{~d} t}=\Pi-\Phi
$$

where $\Phi$ is the entropy flux rate from the system to the environment and $\Pi$ is the entropy production rate, which is always non-negative. In the steady-state $d S / d t=0$ but this does not mean that the system is in equilibrium. Instead, it only implies that $\Pi=\Phi$. The case $\Pi \neq 0$ and $\mathrm{d} S / \mathrm{d} t=0$ characterizes a non-equilibrium steady-state (NESS), where all the entropy that is constantly being produced is flowing to the environment. Only in the particular case of thermal equilibrium should one get $\Pi=\Phi=0$. The standard formulation of the entropy production in open quantum dynamics, as described by a master equation, uses the von Neumann entropy as the basic underlying quantity. In this case, it can be shown [39, 40] that the associated entropy 
production rate is given by

$$
\Pi_{\mathrm{vN}}=-\partial_{t} \operatorname{tr}\left\{\rho \ln \rho-\rho \ln \rho_{\mathrm{eq}}\right\}
$$

where $\rho_{\mathrm{th}}=e^{-\beta H} / Z$ is the thermal fixed point of the Linbdlad equation. This results in a flux rate

$$
\Phi_{\mathrm{vN}}(t)=-\frac{1}{T} \operatorname{tr}[H \mathcal{D}(\rho)]
$$

where $\mathcal{D}$ is a dissipatior in a Lindblad equation.

\subsection{Wigner entropy production}

Eqs. 4.22 and 4.23 diverge in the limit $T \rightarrow 0$. The divergence in the latter is trivial, whereas that in the former is related to the fact that as $T \rightarrow 0$ the reference state $\rho_{\text {th }}$ tends to a pure state (the relative entropy can diverge when the support of $\rho$ is not contained in that of $\rho_{\text {th }}$ ). This divergence, which is sometimes referred to as the "zero temperature catastrophe" and is a peculiar feature of quantum systems. It is not directly related to the third law of thermodynamics, as there are several systems (e.g. in quantum optics and atomic physics) which are well described by zero temperature baths. Notice also that $d S / d t$ is always finite, so that the divergences of $\Pi$ and $\Phi$ should cancel out in eq. 4.21

In order to shed light on this question, in ref. [37] a definition for Wigner entropy production was proposed that satisfies the proprieties described above. The definition is analogous to the von Neumann case, but instead of considering the Lindblad equation, one considers the FokkerPlank equations, obtaining

$$
\begin{aligned}
& \Pi=-\int \mathrm{d}^{2} \alpha \mathcal{D}(W) \ln \left(W / W_{\mathrm{eq}}\right), \\
& \Phi=\int \mathrm{d}^{2} \alpha \mathcal{D}(W) \ln W_{\mathrm{eq}} .
\end{aligned}
$$

In particular the entropy flux for a single harmonic oscillator can be written as

$$
\Phi=\frac{\gamma}{\bar{n}+1 / 2}\left(\left\langle a^{\dagger} a\right\rangle-\bar{n}\right)
$$


This quantity is now always finite, even at $T=0(\bar{n}=0)$. Moreover, when $T$ is large we can approximate $\omega(\bar{n}+1 / 2) \simeq T$, in which case we recover the von Neumann result in eq. 4.22 . This in turn can be generalized in the case of multiple modes coupled to $k$ reservoirs

$$
\Phi=\sum_{k} \frac{\gamma_{k}}{\bar{n}_{k}+1 / 2}\left(\left\langle a_{k}^{\dagger} a_{k}\right\rangle-\bar{n}_{k}\right)
$$

where $a_{k}$ only counts the modes that couple to reservoirs.

Though it is possible to generalize the concept of entropy production for multiple environments a shortcut can be taken in the case of the NESS. By realizing that $\frac{\mathrm{d} S}{\mathrm{~d} t}=0$ this can be used to evaluate the entropy production. 


\section{Chapter 5}

\section{Thermodynamics and information in a}

\section{bosonic chain}

In this section the theory of Wigner entropy described in the previous chapter will be applied to a concrete transport problem: a Bosonic chain where the first and last modes are coupled to an environment with the Lindblad equation

$$
\frac{d \rho}{d t}=\mathcal{L} \rho=-i[H, \rho]+\mathcal{L}_{1} \rho+\mathcal{L}_{N} \rho
$$

where

$$
\mathcal{L}_{i} \rho \equiv \gamma_{i}\left(\bar{n}_{i}+1\right) \mathrm{D}\left[a_{i}, a_{i}^{\dagger}, \rho\right]+\gamma_{i}\left(\bar{n}_{i}\right) \mathrm{D}\left[a_{i}, a_{i}^{\dagger}, \rho\right]
$$

and

$$
H=\sum_{j=1}^{N} \omega a_{j}^{\dagger} a_{j}+V \sum_{j=1}^{N-1} a_{j}^{\dagger} a_{j+1}+a_{j+1}^{\dagger} a_{j} .
$$

\subsection{A note on global vs. local master equations}

The Lindblad term in the equation above is not what would be obtained with the general master equation derivations described in chapter 2 . In that case, when decomposing the systemenvironment interaction in eigenoperators a combination of all modes from the system would be obtained, regardless of the fact that the environment is only coupled to the first and last modes, which would result in what is known as a global master equation. The approach taken 
in this section is what is known as phenomenological (or local) approach, where the dissipator originated from a microscopic derivation ignoring the system-system interactions (or alternatively considering only one mode). It might seem intuitive (or even obvious) that the global approach is better, but that is still a current topic of discussion in the open quantum system community, with both approaches having problems [41]. In particular, the global approach does not conserve heat currents inside the chain, which makes it the less favorable one when studying transport.

\subsection{NESS}

In ref. [42] the NESS of a Bosonic chain with the Lindblad equation as in eq. 5.1 was completely characterized in what will be refered as the reduced covariace matrix $[C]_{i, j} \equiv\left\langle a_{i}^{\dagger} a_{j}\right\rangle+\frac{1}{2} \delta_{i j}$ resulting in

$$
\begin{gathered}
C=\left(\bar{n}+\frac{1}{2}\right) \mathbb{1}+\Delta n D . \\
\bar{n}=\frac{n_{1}+n_{N}}{2} \quad \Delta n=\frac{n_{1}-n_{N}}{2}
\end{gathered}
$$

where

$$
\begin{aligned}
& D=\left[\begin{array}{cccccc}
e_{1} & x & & & & \\
x^{*} & e_{m} & x & & & \\
& \ddots & \ddots & \ddots & & \\
& & & x^{*} & e_{m} & x \\
& & & & x^{*} & e_{N}
\end{array}\right] \\
& e_{1}=\frac{\gamma^{2}}{4 V^{2}+\gamma^{2}}=-e_{N} \quad e_{m}=0 \quad x=-i \frac{2 V \gamma}{4 V^{2}+\gamma^{2}} \text {. }
\end{aligned}
$$

This was studied in the context of heat current and transport, which is a sensitive topic in the discussion of local vs. global equation. In particular this model exhibits ballistic heat transport, which can be noted from the fact that the effective temperature of all modes (except the first and the last) is the same. 


\subsection{Notes on the reduced covariance matrix}

At the time we were studying this problem, all the theory of Wigner entropy was built for the covariance matrix $\Theta$ defined previously, but, in some situations, the only correlations that matter are those of the form $\left.\left\langle a_{i}^{\dagger} a_{j}\right\rangle\right\rangle_{1}^{1}$, as is the case for this Bosonic chain.

Because of this, it is necessary to understand the relation between $\Theta$ and $C$, that is given by equations $5.5,5.6$ and 5.7 .

$$
\begin{gathered}
\Theta=C \otimes\left(\begin{array}{ll}
0 & 0 \\
0 & 1
\end{array}\right)+C^{\top} \otimes\left(\begin{array}{ll}
1 & 0 \\
0 & 0
\end{array}\right), \\
\Theta^{-1}=C^{-1} \otimes\left(\begin{array}{ll}
0 & 0 \\
0 & 1
\end{array}\right)+\left(C^{\top}\right)^{-1} \otimes\left(\begin{array}{ll}
1 & 0 \\
0 & 0
\end{array}\right), \\
|\Theta|=|C|^{2} .
\end{gathered}
$$

With these relations it is possible to calculate the total entropy production and flux using the reduced covariance matrix. For example

$$
\frac{\mathrm{d} S}{\mathrm{~d} t}=\operatorname{tr}\left\{C^{-1} \frac{\mathrm{d} C}{\mathrm{~d} t}\right\}
$$

The Wigner functions in terms of $\Theta$ is given by

$$
W=\frac{1}{\pi^{N}|\Theta|^{\frac{1}{2}}} \exp \left\{-\frac{1}{2} \xi^{\dagger} \Theta^{-1} \xi\right\}
$$

where $\xi^{T}=\left(\alpha_{1}, \alpha_{1}^{*}, \alpha_{2}, \alpha_{2}^{*} \ldots\right)$. In order to write $\Theta$ in terms of $\mathrm{C}$ we first define a vector $a^{T}=$ $\left(\alpha_{1}, \alpha_{2} \ldots\right)$ and note the following properties

$$
\xi=a \otimes\left(\begin{array}{l}
1 \\
0
\end{array}\right)+a^{*} \otimes\left(\begin{array}{l}
0 \\
1
\end{array}\right)
$$

Rewriting the Wigner function we get

$$
W=\frac{1}{\pi^{N}|C|} \exp \left\{-a^{\dagger} C^{-1} a\right\}
$$

\footnotetext{
${ }^{1}$ when there is no squeezing for example
} 
The mutual information for the reduced covariace matrix is given by

$$
\mathcal{I}_{\mathcal{A B}}=\frac{1}{2} \ln \frac{\left|\Theta_{A}\right|\left|\Theta_{B}\right|}{|\Theta|_{A B}}=\ln \frac{\left|C_{A}\right|\left|C_{B}\right|}{|C|_{A B}} .
$$

\subsection{Tridiagonal Toeplitz matrix and Chebishev Polynomials}

The Rényi-2 entropy depends on the determinant of the covariance matrix, which is almost a tridiagonal Toeplitz matrix. For that reason it is useful to calculate the determinant of such matrices in order to generalize the result for the covariance matrix of the NESS. The determinant of a tridiagonal Toeplitz matrix of the form

$$
T=\left[\begin{array}{cccccc}
\frac{a}{y} & i & & & & \\
-i & \frac{a}{y} & i & & & \\
& \ddots & \ddots & \ddots & & \\
& & & -i & \frac{a}{y} & i \\
& & & & -i & \frac{a}{y}
\end{array}\right]
$$

can be obtained by the recursion relation that defines determinants, given by

$$
\begin{aligned}
& f_{n}=\frac{a}{y} f_{n-1}+f_{n-2} \\
& f_{1}=\frac{a}{y} \\
& f_{0}=1
\end{aligned}
$$

The Chebishev polynomials $C_{n}(x)$, where $x=\frac{a}{2 y}$ satisfy (or are defined by) this relation as well. It would be interesting to study a chain in the thermodynamic limit, which implies $n>>1$. There is an useful and exact representation of these polynomials given by

$$
\begin{aligned}
& C_{n}(\cosh \theta)=\frac{\sinh (n+1) \theta}{\sinh \theta} \\
& \theta=\operatorname{arccosh} \frac{a}{2 y}
\end{aligned}
$$


In this form it is straightforward to calculate the determinant assuming $n>>1$.

$$
\begin{aligned}
& C_{n>>1}(\cosh \theta) \approx \frac{\frac{\exp \{n \theta\}}{2} \sinh \theta+\frac{\exp \{n \theta\}}{2} \cosh \theta}{\sinh \theta} \\
& \theta=\operatorname{arccosh} \frac{a}{2 y}
\end{aligned}
$$

\subsection{Covariance like, toeplitz like matrix determinant}

In order to calculate minors or the determinant of the covariance matrix it is necessary to calculate the determinant of the following matrix

$$
T_{C}=y \times\left[\begin{array}{cccccc}
\frac{a_{1}}{y} & i & & & & \\
-i & \frac{a}{y} & i & & & \\
& \ddots & \ddots & \ddots & & \\
& & & -i & \frac{a}{y} & i \\
& & & & -i & \frac{a_{n}}{y}
\end{array}\right]
$$

which can be done by the recursive definition of the determinant and the property for $n \times n$ matrices $M$ that $|k M|=k^{n}|M|$. For a $n \times n$ matrix we have

$$
\left|T_{C n \times n}\right|=y^{n} f_{n-4}-y^{n-1}\left(a_{1}+a_{n}\right) f_{n-3}+a_{1} a_{n} y^{n-2} f_{n-2} .
$$

\subsection{Mutual information and entropy production}

The previous formula can be used to calculate the determinant and minors of the reduced covariance matrix, and then entropy production and mutual information.

The mutual information can be calculated using equations 5.12 and 5.20 and is given by

$$
\mathcal{I}_{\mathcal{A B}}=\ln \left(\frac{1}{2}+\frac{a}{2} \frac{1}{\sqrt{a^{2}-4 y^{2}}}\right)
$$

Note that, in the thermodynamic limit it does not matter which parts of the chain you are comparing to get the mutual information, as long as both sections are also in the thermodynamic limit. 
The entropy production can also be evaluted to be

$$
\Pi=\frac{4 V^{2} \gamma \Delta n}{4 V^{2}+\gamma^{2}}\left(\frac{1}{n_{N}+1 / 2}-\frac{1}{n_{1}+1 / 2}\right) .
$$

It is interesting to note that in the case $\Delta n=0$, which would be the case where the system thermalizes, the entropy production is zero, which is expected from the definition. Symmilar results have been obtained in ref. [43]. 


\section{Chapter 6}

\section{Quantum Darwinism}

The quantum to classical transition is a major problem in physics. Though this is not a solved problem, quantum Darwinism is a theory that explains the emergence of a particular classical phenomena: objective realism [44]. In an objective reality it is expected that different observers agree on what they are observing, which is true for classical mechanics (after-all measurement is not even a part of the postulates).

Quantum Darwinism proposes that objective reality is an emergent propriety of a quantum system if when measuring different fractions of the bath the same information about the system can be obtained. In references [45, 46] it was shown that if the time evolution of all small partition of the bath is described by a specific type of map, known as "Measure-and-Prepare (MP) map", then objective reality will be a propriety of the environment.

In this section evidences will be shown that a particular map is MP for a microscopic environment. This is a very different view on open quantum systems from the previous chapters, since the environment itself will be studied instead of the system.

\subsection{Model and time evolution}

The system that will be studied consists of an harmonic oscillators, which represents the system, coupled to $N$ other harmonic oscillator through a Hamiltonian of the form

$$
H=\omega a^{\dagger} a+\sum_{k} \Omega_{k} b_{k}^{\dagger} b_{k}+\sum_{k} \gamma_{k}\left(a^{\dagger} b_{k}+b_{k}^{\dagger} a\right)
$$




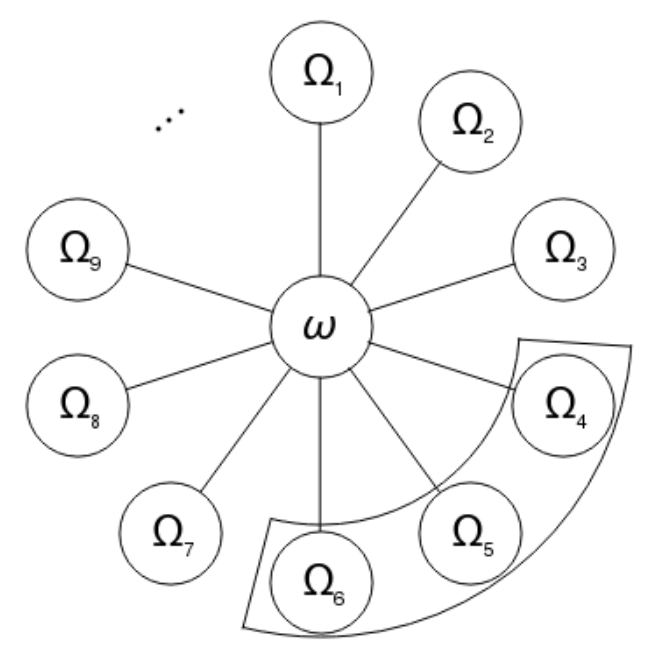

Figure 6.1: visual representation of the model and of a fraction of the bath

Instead of looking at the map on a general state we can apply it to a coherent state. Once that is understood we can apply the map to the decomposition of a generic state in terms of coherent states. Take an initial coherent state of the form:

$$
|\psi\rangle=|\alpha\rangle \prod_{k}\left|\beta_{k}\right\rangle
$$

Since the Hamiltonian is quadratic in the creation and annihilation it preserves Gaussianity, but since it does not have squeezing terms it will take coherent states to coherent states as well. With that in mind the state in time $t$ will be of the form

$$
\begin{gathered}
|\psi(t)\rangle=|g(t)\rangle \prod_{k}\left|f_{k}(t)\right\rangle \\
|g(t)\rangle=e^{-|g|^{2} / 2} e^{g a^{\dagger}}|0\rangle
\end{gathered}
$$

We can now look at Schrödinger's equation for the state, but to do so first we apply the Hamiltonian:

$$
H|\psi(t)\rangle=\left(\omega a^{\dagger} a+\sum_{k} \Omega_{k} b_{k}^{\dagger} b_{k}+\sum_{k} \gamma_{k}\left(a^{\dagger} b_{k}+b_{k}^{\dagger} a\right)\right)|\psi(t)\rangle
$$

But since the states are always coherent states, applying the annihilation operators gives

$$
\begin{aligned}
& \left(\omega a^{\dagger} a+\sum_{k} \Omega_{k} b_{k}^{\dagger} b_{k}+\sum_{k} \gamma_{k}\left(a^{\dagger} b_{k}+b_{k}^{\dagger} a\right)\right)|\psi(t)\rangle= \\
& =\left(\omega g a^{\dagger}+\sum_{k} \Omega_{k} f_{k} b_{k}^{\dagger}+\sum_{k} \gamma_{k}\left(f_{k} a^{\dagger}+g b_{k}^{\dagger}\right)\right)|\psi(t)\rangle
\end{aligned}
$$


Now looking at the time derivative of $|\psi(t)\rangle$, in reference to equation 6.4

$$
\frac{\mathrm{d}|g(t)\rangle}{\mathrm{d} t}=-\frac{1}{2} \frac{\mathrm{d}|g|^{2}}{\mathrm{~d} t}|g\rangle+\dot{g} a^{\dagger}|g\rangle
$$

Applying the derivative to all terms gives

$$
\frac{\mathrm{d}|\psi(t)\rangle}{\mathrm{d} t}=-\frac{1}{2} \frac{\mathrm{d}|g|^{2}}{\mathrm{~d} t}|\psi\rangle+\dot{g} a^{\dagger}|\psi\rangle+\sum_{k}\left(-\frac{1}{2} \frac{\mathrm{d}\left|f_{k}\right|^{2}}{\mathrm{~d} t}|\psi\rangle+\dot{f}_{k} b_{k}^{\dagger}|\psi\rangle\right)
$$

This results in the following set of equations

$$
\begin{aligned}
& \frac{\mathrm{d}}{\mathrm{d} t}\left(|g|^{2}+\sum_{k}\left|f_{k}\right|^{2}\right)=0, \\
& \mathrm{i} \dot{g}=\omega g+\sum_{k} \gamma_{k} f_{k}, \\
& \mathrm{i} \dot{f}_{k}=\Omega_{k}+\gamma_{k} g, \\
& g(0)=\alpha, \\
& f_{k}(0)=\beta_{k} .
\end{aligned}
$$

We can rewrite equations 6.13 conveniently in matrix form

$$
\begin{gathered}
M=\left(\begin{array}{cccc}
\omega & \gamma_{1} & \gamma_{2} & \ldots \\
\gamma_{1} & \Omega_{1} & 0 & \ldots \\
\gamma_{2} & 0 & \Omega_{2} & \ldots \\
\vdots & \vdots & \vdots & \ddots
\end{array}\right) \\
x(t)=e^{\mathrm{i} M t} x(0):=U(t) x(0)
\end{gathered}
$$

where $x=\left(g, f_{1}, f_{2}, \ldots, f_{N}\right)$.

This results in a Schrödinger's equation for a $N+1$ level system, which is reasonable since coherent states are much less complex than the whole Hilbert space. This also means the system's complexity does not scale exponentially as it usually does with many-body systems. But of course we are not only interested in coherent states, so we look at a coherent state decomposition of our initial state, which would give the following density matrix in time 


$$
\rho_{S B}=\int \mathrm{d}^{2} \alpha \mathrm{d}^{2} \boldsymbol{\beta} P_{S}(\alpha) P_{B}(\boldsymbol{\beta})\left|g_{t}(\alpha, \boldsymbol{\beta})\right\rangle\left\langle g_{t}(\alpha, \boldsymbol{\beta})\left|\prod_{k}\right| f_{k t}(\alpha, \boldsymbol{\beta})\right\rangle\left\langle f_{k t}(\alpha, \boldsymbol{\beta})\right|
$$

where $\left|g_{t}(\alpha, \boldsymbol{\beta})\right\rangle$ is now defined as the solution of equations 6.13 in time $t$ with initial conditions $\alpha, \boldsymbol{\beta}$. Note that the system and bath are assumed to be initially non-correlated(the $P$ functions are factored). A generic initial state can always be decomposed in coherent states using the $P$ representation [38]. As an example, the thermal state's $\mathrm{P}$ function is given by

$$
P_{B}(\beta)=\prod_{k} \frac{e^{-\left|\beta_{k}\right|^{2} / \bar{n}_{k}}}{\pi \bar{n}_{k}}
$$

The reason why the $P$ representation was used is that it allows partial traces to be taken conveniently. For instance, tracing over the system $S$, as well as a part $b$ of the entire environment $B$, we get,

$$
\rho_{b}=\operatorname{tr}_{s, B / b} \rho_{S B}(t)=\int \mathrm{d}^{2} \alpha \mathrm{d}^{2} \boldsymbol{\beta} P_{S}(\alpha) P_{B}(\boldsymbol{\beta}) \prod_{k \in b}\left|f_{k t}(\alpha, \boldsymbol{\beta})\right\rangle\left\langle f_{k t}(\alpha, \boldsymbol{\beta})\right|,
$$

which is useful since we are interested in partitions of the bath.

\subsection{Measure-and-prepare maps}

Measure-and-prepare maps are formally defined as

$$
\mathcal{E}_{b}\left(\rho_{S}\right)=\sum_{k} \operatorname{tr}\left\{M_{k} \rho_{S}\right\} \sigma_{k b}
$$

or in the continuous case

$$
\mathcal{E}_{b}\left(\rho_{S}\right)=\int \mathrm{d}^{2} \alpha \operatorname{tr}\left\{M_{\alpha} \rho_{S}\right\} \sigma_{\alpha b}
$$

where $M_{k}$ is a POVM as stated in chapter 2. The name MP comes from the fact that the output $\mathcal{E}_{b}\left(\rho_{S}\right)$ is prepared by combining $\sigma_{k b}$ weighted by the results of the measurement $\operatorname{tr}\left\{M_{k} \rho_{S}\right\}$ on $\rho_{S}$. 
The idea now is to fix the bath's initial state as a thermal state to then interpret 6.18 as a map

$$
\begin{aligned}
\Lambda_{b} & : \mathcal{L}_{S} \rightarrow \mathcal{L}_{b}, \\
\Lambda_{b}\left(\rho_{S}\right) & =\int \mathrm{d}^{2} \alpha \mathrm{d}^{2} \boldsymbol{\beta} P_{S}(\alpha) P_{B}(\boldsymbol{\beta}) \prod_{k \in b}\left|f_{k}(\alpha, \boldsymbol{\beta})\right\rangle\left\langle f_{k}(\alpha, \boldsymbol{\beta})\right| .
\end{aligned}
$$

At this stage it is not clear at all that this is a measure-and-prepare map.

In order to simplify the results it is possible to integrate the $P$ function of the environment if it is assumed to be a known state, such as a thermal state. By first expanding the coherent states in displacement operators to separate the operator part from the $\beta$ dependent part and also with the time evolution of $f$

$$
\begin{gathered}
|\alpha\rangle\langle\alpha|=\int \mathrm{d}^{2} \lambda e^{-|\lambda|^{2} / 2} e^{\lambda \alpha^{*}-\alpha^{*} \lambda} D^{\dagger}(\lambda), \\
f_{k}(\alpha, \beta)=U_{k 0} \alpha+\sum_{q} U_{k q} \beta_{q}
\end{gathered}
$$

it is possible to write the map as

$$
\Lambda_{b}\left(\rho_{S}\right)=\int \mathrm{d}^{2} \alpha \mathrm{d}_{b}^{2} v P_{S}(\alpha)\left[\prod_{k \in b} D_{k}^{\dagger}\left(v_{k}\right)\right] \exp \left\{\left[-v^{\dagger} C \nu+\sum_{k \in b} U_{k 0}^{*} \alpha^{*} v_{k}-U_{k 0} \alpha v_{k}^{*}\right]\right\}
$$

where $d_{b}^{2} v=\prod_{k \in b} \mathrm{~d}^{2} v_{k}$ and

$$
C_{k l}=\sum_{q=1}^{N} \bar{n}_{q} U_{k q} U_{l q}^{*}+\frac{\delta_{k l}}{2}
$$

In order to get closer to obtaining a POVM it is reasonable use the $Q$ representation, which is defined as

$$
Q_{\rho}(\alpha)=\langle\alpha|\rho| \alpha\rangle=\operatorname{tr}\left\{M_{\alpha} \rho\right\}
$$

where $M_{\alpha}=|\alpha\rangle\langle\alpha|$ is a POVM due to the completeness-like relation of coherent states. Using

$$
P(\alpha)=\int \frac{\mathrm{d}^{2} \lambda \mathrm{d}^{2} \gamma}{\pi} e^{|\lambda|^{2}} e^{(\alpha-\gamma) \lambda^{*}-\left(\alpha-\gamma^{*}\right) \lambda} Q(\gamma)
$$


results in

$$
\begin{aligned}
\Lambda_{b}\left(\rho_{s}\right) & =\int \mathrm{d}^{2} \gamma Q(\gamma) \sigma_{\gamma b}, \\
\sigma_{\gamma b} & =\int \mathrm{d}_{b}^{2} v\left[\prod_{k \in b} D_{k}^{\dagger}\left(v_{k}\right)\right] \chi_{\gamma}(v), \\
\chi_{\alpha}(v) & =\exp \left\{-\sum_{k l \in b} \tilde{C}_{k l} v_{k}^{*} v_{l}+\sum_{k \in b}\left(U_{k 0}^{*} \alpha^{*} v_{k}-U_{k 0} \alpha v_{k}\right)\right\}, \\
\tilde{C}_{k l} & =C_{k l}-U_{k 0} U_{l 0}^{*} .
\end{aligned}
$$

This map is almost a measure-and-prepare except that it is not obvious that $\sigma$ are states. Note that if $\tilde{C}$ is a covariance matrix then $\sigma$ are Gaussian states. In fact they are state iff $\tilde{C}$ is a valid covariance matrix. A covariance matrix is valid if satisfies the bona-fide relation

$$
\tilde{C}-\frac{I}{2} \geq 0
$$

\subsection{Numerical analysis of the problem}

This section is dedicated to a numerical study of the proposed map for an ohmic spectral density. In this case, there is evidence that the previously proposed map is always a measure-and-prepare map for any partition size and any temperature greater than zero, given a large enough bath.

\subsection{Bath modeling}

Since the bath is expected to be large, even for long times we expect it to be approximately thermal, meaning, if the system is traced out the state that will be obtained will be a thermal state plus some small contribution. If that is true then the map from system to environment (or for a fraction of the environment) is close to a measure-and-prepare map in a trivial sense, where the $\sigma$ matrices in eq. 6.19 would just be thermal states. This will be a key point in the argument that the following map will always be a measure-and-prepare map for large finite baths. 


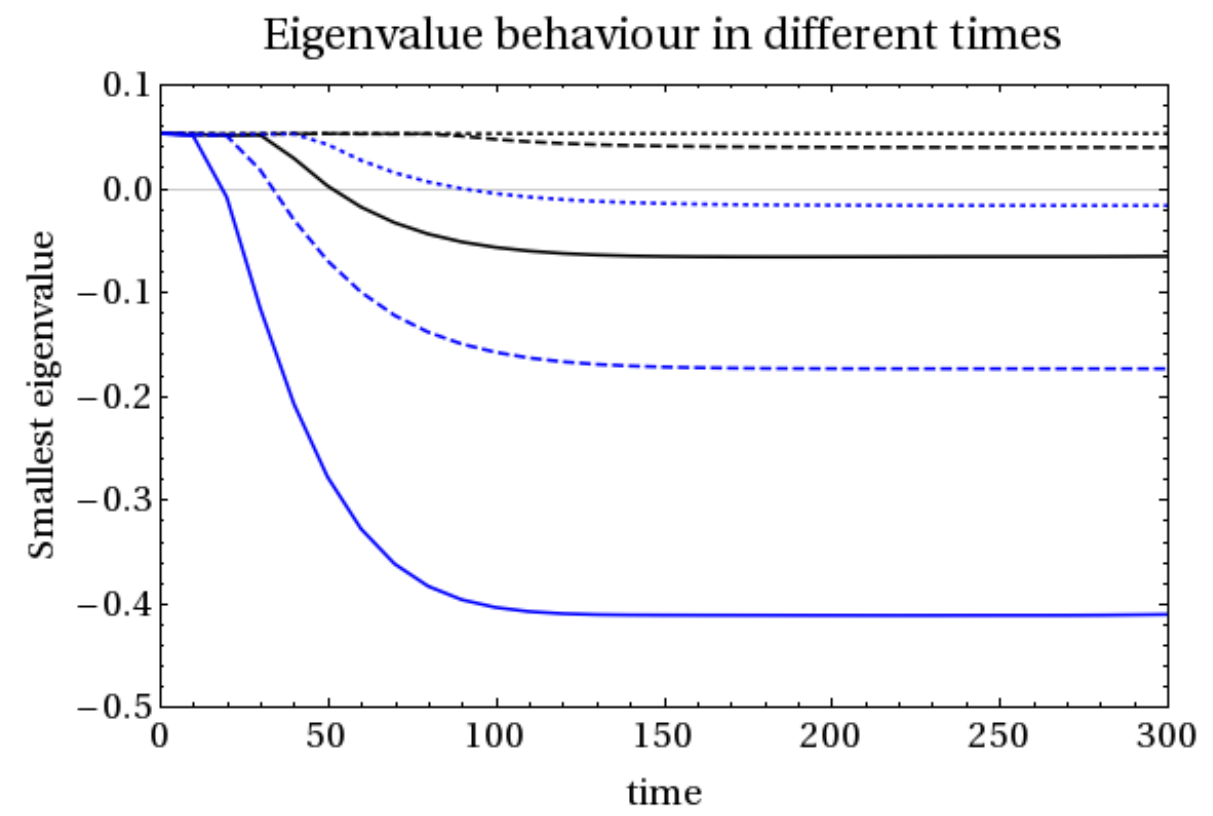

Figure 6.2: bona-fide test for partitions sizes 1(black) and 3(blue) for $\mathrm{N}=50$ (line),100(dashed) and 200(dotted)

By choosing

$$
\begin{array}{r}
\Omega_{k}=1-\Delta \omega+\frac{2 \Delta \omega(k-1)}{N-1} \\
\gamma_{k}=\frac{\gamma}{\sqrt{N}}
\end{array}
$$

with $\gamma \ll \Delta \omega$ this reproduces an Ohmic spectral density when $N \rightarrow \infty$. This is a general method of obtaining a spectral density: uniform frequency distribution plus the desired spectral density discretized in the interactions.

The numerical values used were $\omega=1, \Delta \omega=0.5$ and $\gamma=0.1$. This was verified to approximate the ohmic spectral density well enough for the purposes of this dissertation.

\subsection{Bona-fide tests}

Using the finite bath from the previous session we can construct a matrix as in equation 6.33 . To verify if that is positive we look at the smallest eigenvalue. If that is positive, then $\tilde{C}$ is a valid covariance matrix and the map is measure-and-prepare.

Figure 2 shows the smallest eigenvalue of $\tilde{C}-I / 2$ as a function of time for two different partition sizes and for varying bath size. The behavior seems to be monotonically decreasing with time, and monotonically increasing with bath size. If this map does converge to a thermal 


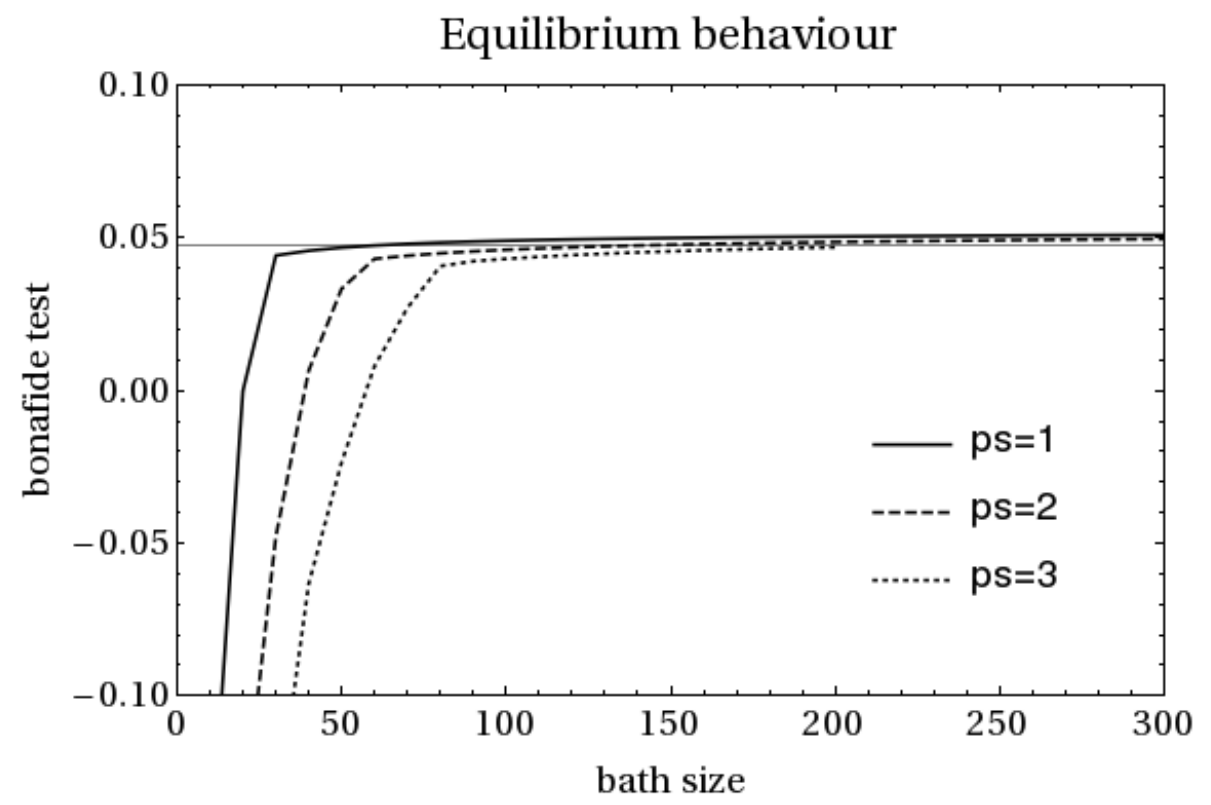

Figure 6.3: visual representation that the system has a predictable minimum eigenvalue which is positive for large $\mathrm{N}$, being a strong indication that the map is measure-and-prepare for finite baths

state then this is a strong evidence that for a large $N$ this map is always measure-and-prepare, because being monotonically decreasing would guarantee that $\min [\operatorname{eig}(\tilde{C})] \geq \min \left[\operatorname{eig}\left(\rho^{t h}\right)\right] \geq 0$. In this case we could predict what would be the smallest eigenvalue after the system converges for large baths, which would be given by

$$
\min \left[\operatorname{eig}\left(\rho^{\text {th }}\right)\right]=\bar{n}(\omega+\Delta \omega) \beta_{B}
$$

for any partition size.

This can be checked by looking only at points after the system thermalizes. In figure 3 the predicted eigenvalue is the grid-line, and for 3 different partition sizes the result seems to match well, being only strictly bigger than 6.36 .

\subsection{Conclusions and perspectives}

Studying explicit maps such as the one presented may give insights in how classicality emerges. A possible continuation of these results would be to prove mathematically that there is an $N<\infty$ where this map is always measure-and-prepare. Another option would be to study how nonmarkovianity affects this approach, since some results indicate that non-markovianity hinders quantum Darwinism[47]. It is also important to point out that a similar map (without the pre- 
trace RWA) was shown analytically to be MP[48]. 


\section{Chapter 7}

\section{Non-equilibrium Green's functions}

Green's function are a powerful tool to study both dynamics and steady states of many-body systems [49]. It is widely used in solid state physics. In this chapter a study on Green's function from a quantum information perspective is made. A similar approach was taken in ref. [50].

\subsection{Model}

The Hamiltonian will be given by

$$
H=\sum_{i j} h_{i j} c_{i}^{\dagger} c_{j}+\sum_{i} \int \mathrm{d} \omega\left(g_{i}(\omega) c_{i}^{\dagger} \eta_{i}(\omega)+\text { h.c. }\right)+\sum_{i} \int \mathrm{d} \omega\left(\omega \eta_{i}^{\dagger}(\omega) \eta_{i}(\omega)\right)
$$

where $c(\eta)$ is the Fermionic annihilation operator for the system (environment) and $h$ is a matrix that defines the network structure of the Fermionic system, a Hamiltonian of sorts. In the case of a chain it is a tridiagonal Toeplitz matrix.

The equations of motion in the Heisenberg picture for a time independent $t^{1}$ operator is given by

$$
\dot{A(t)}=\mathrm{i}[H, A(t)]
$$

\footnotetext{
${ }^{1}$ in the Schrödinger picture
} 
which results in

$$
\begin{aligned}
\dot{c}_{i}(t) & =-\mathrm{i} \sum_{j} h_{i j} c_{j}(t)-\mathrm{i} \int \mathrm{d} \omega g_{i}(\omega) \eta_{i}(\omega ; t), \\
\dot{\eta}_{i}(\omega ; t) & =-\mathrm{i} \omega \eta_{i}(\omega ; t)-\mathrm{i} g_{i}^{*}(\omega) c_{i}(t) .
\end{aligned}
$$

Equation 7.4 can be formally integrated to obtain

$$
\eta_{i}(\omega ; t)=e^{-\mathrm{i} \omega\left(t-t_{0}\right)} \eta_{i}\left(\omega ; t_{0}\right)-\mathrm{i} g_{i}^{*}(\omega) \int_{t_{0}}^{t} \mathrm{~d} s e^{-\mathrm{i} \omega(t-s)} c_{i}(s) .
$$

This in turn can be inserted in eq. 7.3 to obtain the Langevin equation

$$
\dot{c}_{i}(t)=-\mathrm{i} \sum_{j} h_{i j} c_{j}(t)+\xi_{i}(t)-\int_{t_{0}}^{t} \mathrm{~d} s \chi_{i}(t-s) c_{i}(s),
$$

where $\xi$ is known as the noise operator

$$
\xi_{i}(t)=-\mathrm{i} \int \mathrm{d} \omega g_{i}(\omega) e^{-\mathrm{i} \omega\left(t-t_{0}\right)} \eta_{i}\left(\omega ; t_{0}\right)
$$

since it only depends on the bath operators in time zero, and not at all in the system's Hamiltonian, and $\chi$ the memory kernel

$$
\chi_{i}(t-s)=\int \mathrm{d} \omega g_{i}^{*}(\omega) g_{i}(\omega) e^{-\mathrm{i} \omega(t-s)}
$$

since it makes $c_{i}(s)$ explicitly depend on its history.

It is particularly useful to define the spectral density as

$$
\mathcal{J}_{i}(\omega)=2 \pi\left|g_{i}(\omega)\right|^{2}
$$

which implies the memory kernel is the Fourier transform of the spectral density.

In particular, the noise operator has the interesting propriety that

$$
\begin{aligned}
\left\{\xi_{i}(t), \xi_{j}^{\dagger}(s)\right\} & =-\int \mathrm{d} \omega \mathrm{d} v \delta_{i j} g_{i}(\omega) g_{j}^{*}(v) e^{-\mathrm{i} \omega\left(t-t_{0}\right)} e^{\mathrm{i} v\left(s-t_{0}\right)}\left\{\eta\left(\omega ; t_{0}\right), \eta^{\dagger}\left(v ; t_{0}\right)\right\}= \\
& =-\delta_{i j} \int \mathrm{d} \omega \frac{\mathcal{J}_{i}(\omega)}{2 \pi} e^{-\mathrm{i} \omega(t-s)}
\end{aligned}
$$


When the spectral density is constant this simply becomes proportional to a delta in different $t$ 's, in a way that the anti-commuting propriety of the Fermions in frequency or space manifests itself in time for the noise operator ${ }^{2}$

Defining $J_{i}(\omega)=\gamma_{i}$, which implies $\chi_{i}(t-s)=\gamma_{i} \delta(t-s)$ the Langevin equation becomes

$$
\dot{c}_{i}(t)=-\mathrm{i} \sum_{j} h_{i j} c_{j}(t)+\xi_{i}(t)-\gamma_{i} \frac{c_{i}(t)}{2}
$$

where the $1 / 2$ factor in the last term comes from the fact that the integral in time in the memory kernel only includes "half" the delta function. Rewriting eq.7.12 in vector notation

$$
\dot{\boldsymbol{c}}(t)=-W c(t)+\boldsymbol{\xi}(t),
$$

where $W=\mathrm{i} h+\Gamma$ and $\Gamma$ is a diagonal matrix with the vector $\gamma$ as an entry. This equation is now time local, which makes it a lot more tractable.

Eq. 7.13 can in turn be formally integrated with

$$
\boldsymbol{c}(t)=G\left(t-t_{0}\right) \boldsymbol{c}\left(t_{0}\right)+\int_{t_{0}}^{t} \mathrm{~d} s G(t-s) \boldsymbol{\xi}(s)
$$

where $G(t)=\Theta(t) e^{-W t}$, which can be verified by noting that $\dot{G}(t)=\delta(t) e^{-W t}-W G(t)$.

\subsection{Correlations}

There are two types of relevant correlations to calculate: $\left\langle\xi_{i}^{\dagger}(t) \xi_{j}(s)\right\rangle$ and $\left\langle\xi_{i}^{\dagger}(t) c_{j}(s)\right\rangle$.

$$
\left\langle\xi_{i}^{\dagger}(t) \xi_{j}(s)\right\rangle=\int \mathrm{d} \omega \mathrm{d} v g_{i}^{*}(\omega) g_{j}(v) e^{\mathrm{i} \omega\left(t-t_{0}\right)} e^{-\mathrm{i} v\left(s-t_{0}\right)}\left\langle\eta_{i}^{\dagger}\left(\omega ; t_{0}\right) \eta_{j}\left(v ; t_{0}\right)\right\rangle
$$

The appearance of the term $\left\langle\eta_{i}^{\dagger}\left(\omega ; t_{0}\right) \eta_{j}\left(v ; t_{0}\right)\right\rangle$ requires the initial state of the environment to be stated. For a thermal bath $\left\langle\eta_{i}^{\dagger}\left(\omega ; t_{0}\right) \eta_{j}\left(v ; t_{0}\right)\right\rangle=f(\omega) \delta_{i j} \delta(\omega-v)$, resulting in

$$
\left\langle\xi_{i}^{\dagger}(t) \xi_{j}(s)\right\rangle=\delta_{i j} \int \mathrm{d} \omega \frac{\mathcal{J}(\omega)}{2 \pi} e^{\mathrm{i} \omega(t-s)} f_{i}(\omega)
$$

which in the case of a constant spectral density is the Fourier transform of the Fermi-Dirac

\footnotetext{
${ }^{2}$ For example, the same relation (but with commutator) appears for bosons.
} 
distribution.

Using eq. 7.14 and the assumption that the environment is initially uncorrelated with the system

$$
\left\langle\xi_{i}^{\dagger}(t) c_{j}(s)\right\rangle=\int_{t_{0}}^{s} \mathrm{~d} \tau G_{j i}(s-\tau) \int \mathrm{d} \omega \frac{\mathcal{J}_{i}(\omega)}{2 \pi} e^{\mathrm{i} \omega(t-\tau)} f_{i}(\omega)
$$

In the case $\mathcal{J}_{i}(\omega)=\gamma_{i}$

$$
\begin{aligned}
\left\langle\xi_{i}^{\dagger}(t) \xi_{j}(s)\right\rangle & =\delta_{i j} F_{i}(s-t) \\
\left\langle\xi_{i}^{\dagger}(t) c_{j}(s)\right\rangle & =\int_{t_{0}}^{s} \mathrm{~d} \tau G_{j i}(s-\tau) F_{i}(\tau-t)
\end{aligned}
$$

where

$$
F_{i}(t-s)=\frac{\gamma_{i}}{2} \delta(t)+\frac{i \gamma_{i} T_{i}}{2} e^{-i \mu_{i} t} \operatorname{csch}\left(\pi T_{i} t\right)
$$

which sets a characteristic time $\tau_{c} \propto \frac{1}{\pi T}$, after which the correlations between the environment and the system becomes constant. In the next section it will be shown that this implies that the dissipative part depends only on these correlations.

\subsection{Lyapunov equation}

The results obtained in the previous sections allows one to formally calculate the covariance matrix with

$$
\begin{aligned}
C(t) & =G\left(t-t_{0}\right) C_{0} G^{\dagger}\left(t-t_{0}\right)+Z(t) \\
Z(t) & \equiv \int_{t_{0}}^{t} \mathrm{~d} \tau \int_{t_{0}}^{t} \mathrm{~d} \tau^{\prime} G(t-\tau) F\left(\tau-\tau^{\prime}\right) G^{\dagger}\left(t-\tau^{\prime}\right)
\end{aligned}
$$

which in turn can be used to derive the Lyapunov equation for the system

$$
\dot{C}(t)=-W\left(G(t) C_{0} G^{\dagger}(t)\right)-\left(G(t) C_{0} G^{\dagger}(t)\right) W^{\dagger}+\dot{Z}(t)
$$


using $G(t) C_{0} G^{\dagger}(t)=C(t)-Z(t)$

$$
\dot{C}=-\left(W C+C W^{\dagger}\right)+\left[\dot{Z}+W Z+Z W^{\dagger}\right]
$$

$\dot{Z}$ can be evaluate using Leibniz's rule to obtain

$$
\left[\dot{Z}+W Z+Z W^{\dagger}\right] \equiv \mathcal{D}(t)=\int_{0}^{t} \mathrm{~d} \tau\left\{G(\tau) F^{\dagger}(\tau)+F(\tau) G^{\dagger}(\tau)\right\}
$$

It is interesting to note that the matrix elements of the dissipator in the Lyapunov equation depends only on the correlation between the noise operator and the modes in the systems

$$
\mathcal{D}_{i j}(t)=\left\langle\xi_{j}^{\dagger}(t) c_{i}(t)\right\rangle+\left\langle c_{j}^{\dagger}(t) \xi_{i}(t)\right\rangle
$$

\subsubsection{Lindblad equation}

Since most tools to study open quantum system proprieties are readily available for Lindbladlike (by Lindblad-like I mean in Lindblad form but allowing negative Lindblad coefficients) it is useful to understand what dissipator is obtained from $\mathcal{D}$. Since the evolution is Gaussian preserving, the jump operators must be linear in $c$ 's, which makes a fairly specific guess on how the equation must look like

$$
\frac{\mathrm{d} \rho}{\mathrm{d} t}=-\mathrm{i} \sum_{i j}\left[h_{i j} c_{i}^{\dagger} c_{j}, \rho\right]+\sum_{i, j} \gamma_{j i}^{-}(t)\left[c_{i} \rho c_{j}^{\dagger}-\frac{1}{2}\left\{c_{j}^{\dagger} c_{i}, \rho\right\}\right]+\sum_{i j} \gamma_{i j}^{+}(t)\left[c_{i}^{\dagger} \rho c_{j}-\frac{1}{2}\left\{c_{j} c_{i}^{\dagger}, \rho\right\}\right] .
$$

Using the following relations

$$
[A B, C]=A\{B, C\}-\{A, C\} B=A[B, C]+[A, C] B
$$

which can be used to derive

$$
\begin{aligned}
{\left[c_{i}^{\dagger} c_{j}, c_{k}^{(\dagger)}\right] } & =-\delta_{i j} c_{j}\left(\delta_{j k} c_{i}^{\dagger}\right) \\
{\left[c_{i}^{\dagger} c_{j}, c_{g}^{\dagger} c_{k}\right] } & =\delta_{j g} c_{i}^{\dagger} c_{k}-\delta_{i k} c_{g}^{\dagger} c_{j}
\end{aligned}
$$


it is possible to obtain the Lyapunov equation

$$
\begin{gathered}
\frac{\mathrm{d} C}{\mathrm{~d} t}=\tilde{W}(t) C+C \tilde{W}^{\dagger}(t)+\tilde{D}(t) \\
\tilde{W}(t)=\frac{\tilde{\Gamma}}{2}+i \tilde{h}, \quad \tilde{\Gamma}(t)=\gamma^{+}(t)+\gamma^{-}(t) \\
\tilde{D}(t)=\gamma^{+}(t)
\end{gathered}
$$

which allows one to infer that

$$
\begin{array}{r}
\gamma^{+}(t)=D(t) \\
\gamma^{-}(t)=\Gamma-D(t)
\end{array}
$$

\subsection{Bath acting on a single site}

If there is only one environment coupled to, say, the first mode in the system $\mathcal{D}$ will have the form

$$
\mathcal{D}=\left(\begin{array}{cccc}
\mathcal{D}_{11} & \mathcal{D}_{12} & \mathcal{D}_{13} & \ldots \\
\mathcal{D}_{12}^{*} & 0 & 0 & \ldots \\
\mathcal{D}_{13}^{*} & 0 & 0 & \ldots \\
\vdots & \vdots & \vdots & \ddots
\end{array}\right)
$$

which always has only two non-zero eigenvalues,

$$
\lambda^{ \pm}=\frac{1}{2}\left(\mathcal{D}_{11} \pm \sqrt{\mathcal{D}_{11}^{2}+4 \sum_{i>1}\left|\mathcal{D}_{1 i}\right|^{2}}\right)
$$

one positive and one negative. This implies that the system will always be non-Markovian for all times due to eq. 7.34 (except in some limits like infinite temperature, where white noise is obtained). A suitable measure of non-Markovianity in cases like this is the absolute value of the most negative eigenvalue [51]. Though there are Lindblad coefficients that are always negative, this map is guaranteed to be CPTP because it is an exact derivation. 


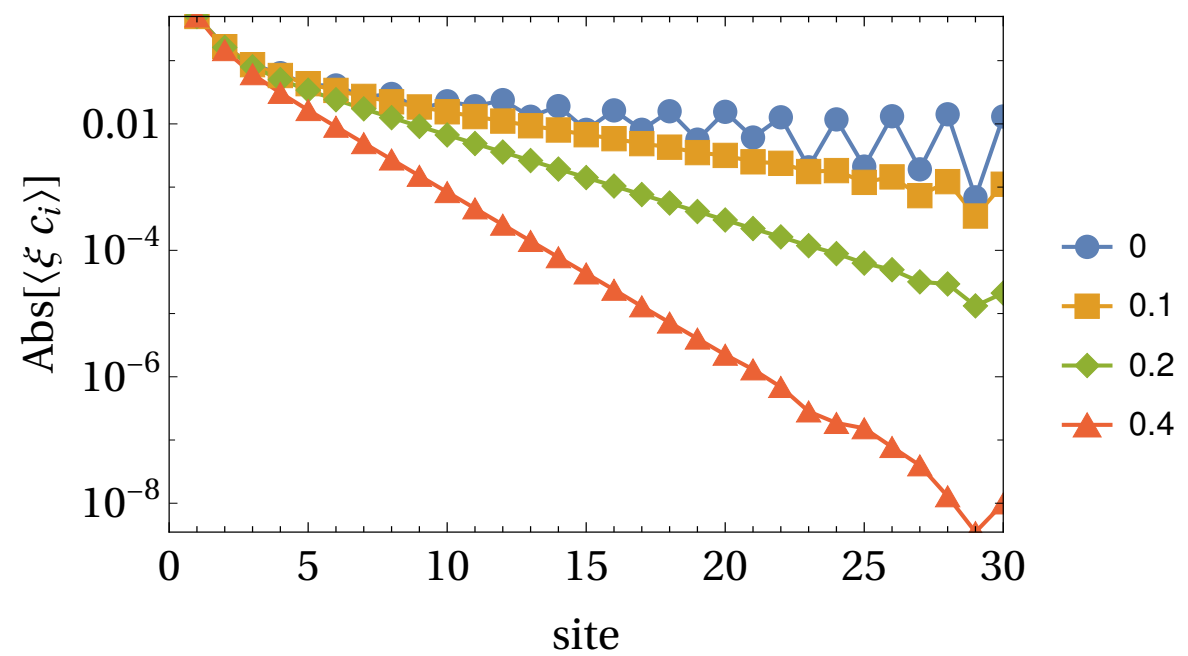

Figure 7.1: Noise-system correlations along the chain, with exponential decay.

\subsubsection{Finite temperature and characteristic distances}

By looking at the dissipator in infinite time some physical characteristics become apparent. In fig. 7.1 it is possible to observe that the system-noise correlation decay exponentially along the chain in finite temperature, which sets a characteristic distance.

This characteristic distance can be evaluated by calculating the inverse of the slope of the logarithmic plot, and can be plotted as a function of temperature as in fig. 7.2. This suggests the characteristic distance is proportional to $T^{-1}$. Note that in the case where the chain has 200 sites (see fig for description) the characteristic distance does not fit perfectly for low temperatures. We believe that is because in such cases the size of the chain is of the order of the characteristic distance in a way that the reflections from the end of the chain become relevant. For that reason a 500 site chain was also considered, where for these temperatures the points fit.

\subsubsection{Time evolution of noise-system correlations}

The dissipator always starts with only the first mode correlated with the environment. Then the correlation starts to propagate along the chain as in fig. 7.3 ,

By looking at the steady state it is possible to infer that this propagation of correlation has constant speed. As seen previously, the temperature sets a characteristic time. It also sets a characteristic distance as can be seen in fig. 7.1. This, and fig. 7.3 are evidences that the propagation has a constant characteristic velocity. It would be interesting to compare this velocity to speed limits of correlation propagations like Lieb-Robinson bounds [52]. 


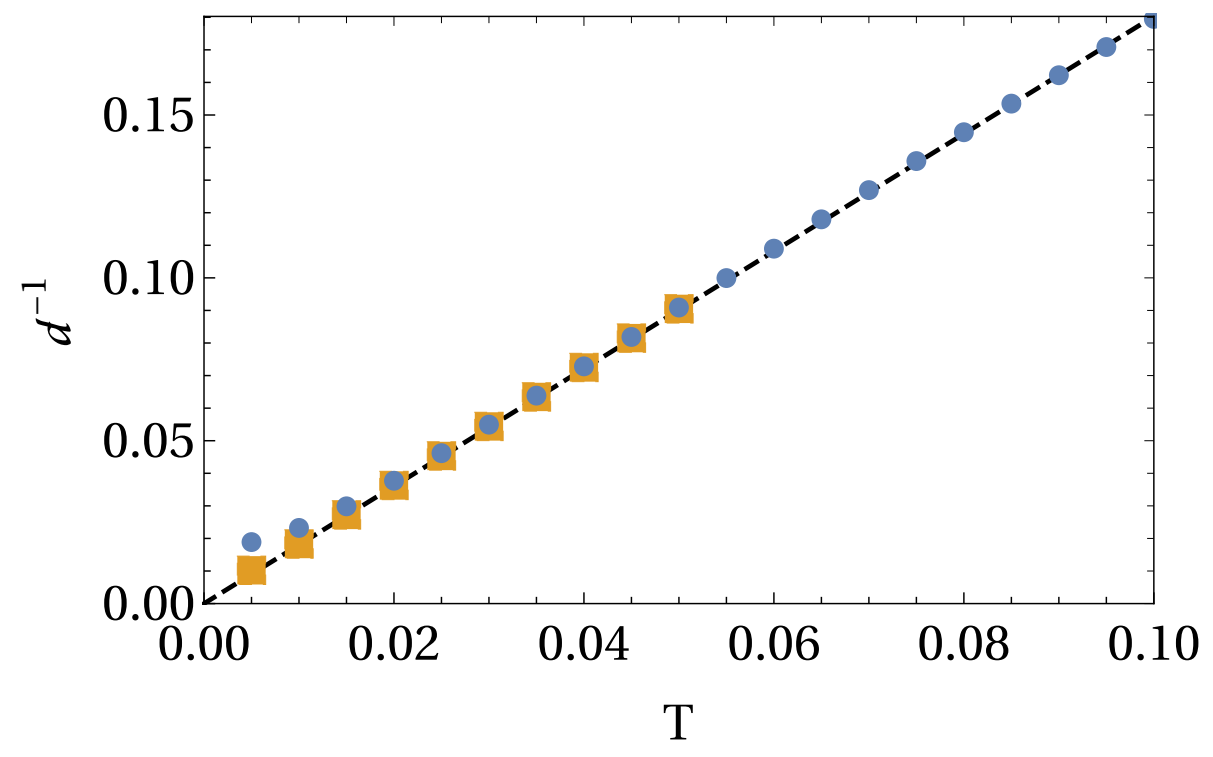

Figure 7.2: Inverse of the characteristic distances for different temperatures and two chain sizes. In blue a 200 site chain and in yellow a 500 site chain.
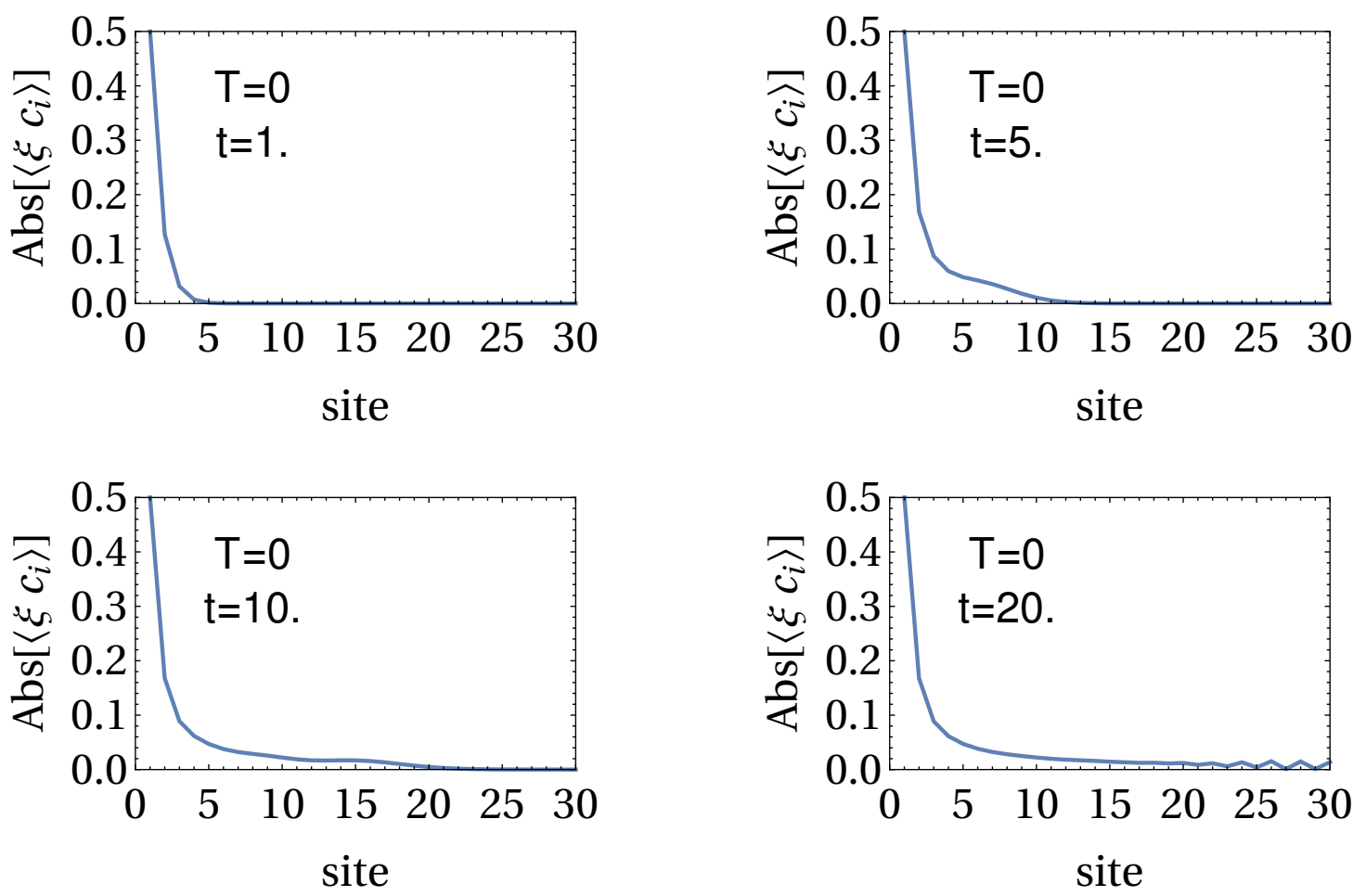

Figure 7.3: Time evolution of system-noise correlation. Note that in the last pane the reflexion due to the fact that the chain is finite can be observed. 


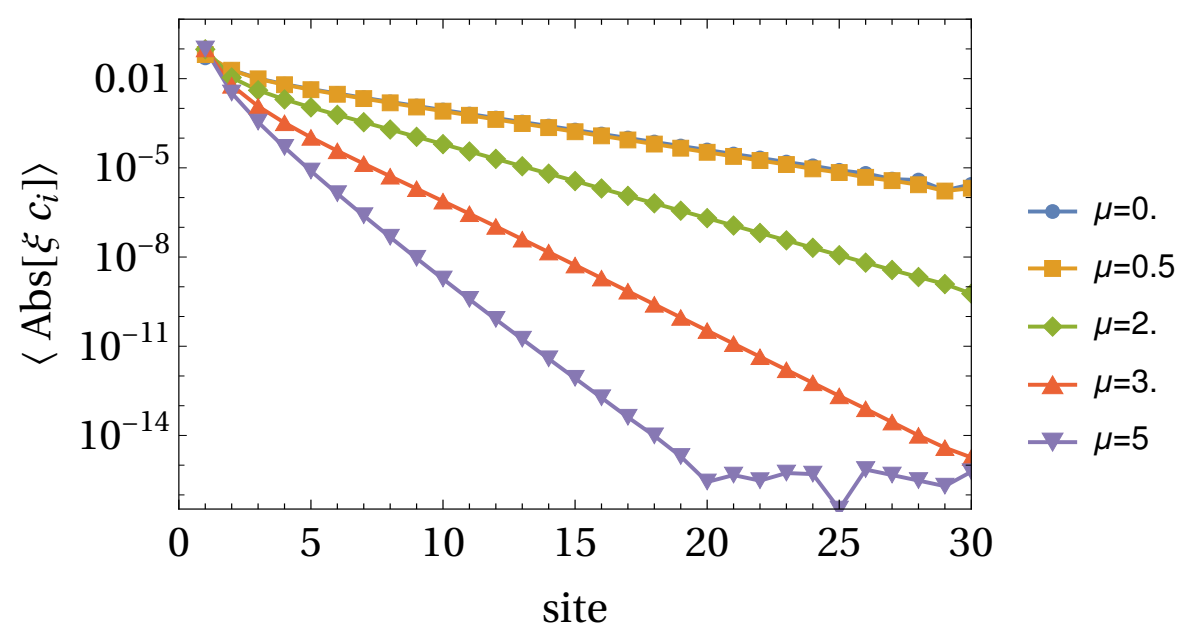

Figure 7.4: System-noise correlations for a fixed temperature $T=0.1$ and varying chemical potentials.

\subsubsection{Infinite time correlations}

It is interesting to note that the characteristic time that the dissipator converges is not particularly relevant to the time it takes for the covariance matrix to converge. If the coupling is weak the covariance matrix converges arbitrarily slower than the dissipator (assuming non-zero temperature, so that the characteristic time does not diverge). In this case, a reasonable approximation for the dynamics would be to consider $\mathcal{D}(t) \approx \mathcal{D}(\infty)$.

It is also interesting to note that in the case $\gamma \ll 1$ the system becomes thermal. In this case $\mathcal{D}(\infty)$ can be calculated by solving the Lyapunov equation with

$$
\mathcal{D}(\infty)=W C_{t h}+C_{t h} W^{\dagger}
$$

\subsubsection{Chemical potential}

The behaviour with chemical potential is also interesting to consider. For example, in the limit of infinite chemical potential the dissipator becomes markovian, and instead of white noise with $\mathcal{D}_{11}=0.5$ we get $\mathcal{D}_{11}=1$, which populates the chain completely in the steady state. The chemical potential also chances the characteristic distances, see fig. 7.4 for example, where the temperature is fixed and the chemical potential varies. 


\subsubsection{Additivity}

One property that would be interesting to consider is if the environments are additive in the sense that adding a new environment simply ads a new single site dissipator as the one considered in this chapter. If that was true it would be trivial to derive the Lyapunov equation, for example assuming that the system thermalizes, so understanding when dissipators are additive can be a powerful tool for microscopic derivations. 


\section{Chapter 8}

\section{Conclusion}

In the context of Holonomic quantum computation it was shown that a zero temperature environment can improve the implementation of a gate assuming a classical error in the implementation time. Some possible optimization were proposed using properties such as nonMarkovianity.

In the context of transport in a Bosonic chain, two entropic quantities, mutual information and entropy production, were calculated, demonstrating the usefulness of Wigner entropy for Gaussian states.

A soluble model was used to study quantum to classical transition using quantum Darwinism, and evidences were shown that said model exhibits objective realism.

Then an exactly soluble Fermionic model was studied, where it was shown that noise-system correlations are linked to dissipation. It would be interesting to study how the characteristic distance behaves with chemical potential, which was done, but no conclusive result was reached like in the case when only temperature is considered. Understanding additivity could also be a powerful tool for a new approach to microscopic derivations, by using the Lyapunov equation to obtain the dissipator. 


\section{Bibliography}

[1] N. G. Van Kampen, Stochastic processes in physics and chemistry, vol. 1. Elsevier, 1992.

[2] V. Gorini, A. Kossakowski, and E. C. G. Sudarshan, "Completely positive dynamical semigroups of n-level systems," Journal of Mathematical Physics, vol. 17, no. 5, pp. 821-825, 1976.

[3] G. Lindblad, "On the generators of quantum dynamical semigroups," Communications in Mathematical Physics, vol. 48, no. 2, pp. 119-130, 1976.

[4] G. W. Ford, J. T. Lewis, and R. F. O'Connell, "Quantum langevin equation,” Phys. Rev. A, vol. 37, pp. 4419-4428, Jun 1988.

[5] K. Mølmer, Y. Castin, and J. Dalibard, "Monte carlo wave-function method in quantum optics," JOSA B, vol. 10, no. 3, pp. 524-538, 1993.

[6] J. Dalibard, Y. Castin, and K. Mølmer, "Wave-function approach to dissipative processes in quantum optics," Physical review letters, vol. 68, no. 5, p. 580, 1992.

[7] M. B. Plenio and P. L. Knight, "The quantum-jump approach to dissipative dynamics in quantum optics," Reviews of Modern Physics, vol. 70, no. 1, p. 101, 1998.

[8] M. B. Plbnio and S. Virmani, "An introduction to entanglement measures," Quantum Info. Comput., vol. 7, pp. 1-51, Jan. 2007.

[9] G. García-Pérez, D. A. Chisholm, M. A. Rossi, G. M. Palma, and S. Maniscalco, "Decoherence without entanglement and quantum darwinism," arXiv preprint arXiv:1907.12447, 2019.

[10] M. A. Nielsen and I. Chuang, "Quantum computation and quantum information,” 2002.

[11] V. Paulsen, Completely bounded maps and operator algebras, vol. 78. Cambridge University Press, 2002.

[12] K. Kraus, States, effects and operations: fundamental notions of quantum theory. Springer, 1983.

[13] M.-D. Choi, “Completely positive linear maps on complex matrices," Linear algebra and its applications, vol. 10, no. 3, pp. 285-290, 1975.

[14] H.-P. Breuer and F. Petruccione, The theory of open quantum systems. Oxford University Press on Demand, 2002. 
[15] H.-P. Breuer, E.-M. Laine, and J. Piilo, "Measure for the degree of non-markovian behavior of quantum processes in open systems," Phys. Rev. Lett., vol. 103, p. 210401, Nov 2009.

[16] R. Zwanzig, "Ensemble method in the theory of irreversibility," The Journal of Chemical Physics, vol. 33, no. 5, pp. 1338-1341, 1960.

[17] S. Nakajima, "On quantum theory of transport phenomena: steady diffusion," Progress of Theoretical Physics, vol. 20, no. 6, pp. 948-959, 1958.

[18] M. O. Scully and M. S. Zubairy, "Quantum optics," 1999.

[19] M. Nakahara, Geometry, topology and physics. CRC Press, 2003.

[20] N. Ramberg and E. Sjöqvist, "Environment-assisted holonomic quantum maps," Physical Review Letters, vol. 122, no. 14, p. 140501, 2019.

[21] R. P. Feynman, "Simulating physics with computers," International journal of theoretical physics, vol. 21, no. 6, pp. 467-488, 1982.

[22] P. W. Shor, "Algorithms for quantum computation: Discrete logarithms and factoring," in Proceedings 35th annual symposium on foundations of computer science, pp. 124-134, Ieee, 1994.

[23] D. A. Lidar and T. A. Brun, Quantum error correction. Cambridge university press, 2013.

[24] J. J. Sakurai, J. Napolitano, et al., Modern quantum mechanics, vol. 185. Pearson Harlow, 2014.

[25] M. V. Berry, "Quantal phase factors accompanying adiabatic changes," Proceedings of the Royal Society of London. A. Mathematical and Physical Sciences, vol. 392, no. 1802, pp. 45-57, 1984.

[26] F. Wilczek and A. Zee, "Appearance of gauge structure in simple dynamical systems," Physical Review Letters, vol. 52, no. 24, p. 2111, 1984.

[27] A. Zee, Quantum field theory in a nutshell, vol. 7. Princeton university press, 2010.

[28] L.-M. Duan, J. I. Cirac, and P. Zoller, "Geometric manipulation of trapped ions for quantum computation,” Science, vol. 292, no. 5522, pp. 1695-1697, 2001.

[29] A. Recati, T. Calarco, P. Zanardi, J. I. Cirac, and P. Zoller, "Holonomic quantum computation with neutral atoms," Physical Review A, vol. 66, no. 3, p. 032309, 2002.

[30] K. Toyoda, K. Uchida, A. Noguchi, S. Haze, and S. Urabe, "Realization of holonomic single-qubit operations," Physical Review A, vol. 87, no. 5, p. 052307, 2013.

[31] J. Anandan, "Non-adiabatic non-abelian geometric phase," Physics Letters A, vol. 133, no. 4-5, pp. 171-175, 1988.

[32] E. Sjöqvist, D.-M. Tong, L. M. Andersson, B. Hessmo, M. Johansson, and K. Singh, "Nonadiabatic holonomic quantum computation," New Journal of Physics, vol. 14, no. 10, p. $103035,2012$. 
[33] Z. Zhang, P. Zhao, T. Wang, L. Xiang, Z. Jia, P. Duan, D. Tong, Y. Yin, and G. Guo, "Single-shot realization of nonadiabatic holonomic gates with a superconducting xmon qutrit," arXiv preprint arXiv:1811.06252, 2018.

[34] M. E. Muller, "A note on a method for generating points uniformly on n-dimensional spheres," Communications of the ACM, vol. 2, no. 4, pp. 19-20, 1959.

[35] A. Serafini, Quantum Continuous Variables: A Primer of Theoretical Methods. CRC Press, 2017.

[36] G. Adesso, D. Girolami, and A. Serafini, "Measuring gaussian quantum information and correlations using the rényi entropy of order 2," Phys. Rev. Lett., vol. 109, p. 190502, Nov 2012.

[37] J. P. Santos, G. T. Landi, and M. Paternostro, "Wigner entropy production rate," Phys. Rev. Lett., vol. 118, p. 220601, Jun 2017.

[38] C. Gardiner, P. Zoller, and P. Zoller, Quantum noise: a handbook of Markovian and non-Markovian quantum stochastic methods with applications to quantum optics, vol. 56. Springer Science \& Business Media, 2004.

[39] H.-P. Breuer, "Quantum jumps and entropy production," Phys. Rev. A, vol. 68, p. 032105, Sep 2003.

[40] H. Spohn, "Entropy production for quantum dynamical semigroups," Journal of Mathematical Physics, vol. 19, no. 5, pp. 1227-1230, 1978.

[41] J. O. González, L. A. Correa, G. Nocerino, J. P. Palao, D. Alonso, and G. Adesso, "Testing the validity of the 'local'and 'global'gkls master equations on an exactly solvable model," Open Systems $\mathcal{E}$ Information Dynamics, vol. 24, no. 04, p. 1740010, 2017.

[42] A. Asadian, D. Manzano, M. Tiersch, and H. J. Briegel, "Heat transport through lattices of quantum harmonic oscillators in arbitrary dimensions," Phys. Rev. E, vol. 87, p. 012109, Jan 2013.

[43] W. T. Malouf, J. P. Santos, L. A. Correa, M. Paternostro, and G. T. Landi, "Wigner entropy production and heat transport in linear quantum lattices," Physical Review A, vol. 99, no. 5, p. 052104, 2019.

[44] W. H. Zurek, "Quantum darwinism," Nature physics, vol. 5, no. 3, p. 181, 2009.

[45] P. A. Knott, T. Tufarelli, M. Piani, and G. Adesso, "Generic emergence of objectivity of observables in infinite dimensions," arXiv preprint arXiv:1802.05719, 2018.

[46] F. G. Brandão, M. Piani, and P. Horodecki, "Generic emergence of classical features in quantum darwinism," Nature communications, vol. 6, p. 7908, 2015.

[47] F. Galve, R. Zambrini, and S. Maniscalco, "Non-markovianity hinders quantum darwinism," Scientific reports, vol. 6, p. 19607, 2016.

[48] J. K. Korbicz, P. Horodecki, and R. Horodecki, "Objectivity in a noisy photonic environment through quantum state information broadcasting," Phys. Rev. Lett., vol. 112, p. 120402, Mar 2014. 
[49] P. Coleman, Introduction to many-body physics. Cambridge University Press, 2015.

[50] M. T. Mitchison and M. B. Plenio, "Non-additive dissipation in open quantum networks out of equilibrium," New Journal of Physics, vol. 20, no. 3, p. 033005, 2018.

[51] A. Rivas, S. F. Huelga, and M. B. Plenio, "Entanglement and non-markovianity of quantum evolutions," Physical review letters, vol. 105, no. 5, p. 050403, 2010.

[52] E. H. Lieb and D. W. Robinson, "The finite group velocity of quantum spin systems," in Statistical mechanics, pp. 425-431, Springer, 1972. 